\title{
Local existence and continuation criteria for solutions of the Einstein-Vlasov-scalar field system with surface symmetry
}

\author{
D. Tegankong ${ }^{1}$; N. Noutchegueme ${ }^{2}$; A.D. Rendall ${ }^{3}$ \\ ${ }^{1}$ Department of Mathematics, Higher Teachers Training College, \\ University of Yaounde 1, Box 47, Yaounde, Cameroon \\ tegankon@uycdc.uninet.cm \\ ${ }^{2}$ Department of Mathematics, Faculty of Sciences, \\ University of Yaounde 1, Box 812, Yaounde, Cameroon \\ nnoutch@uycdc.uninet.cm \\ ${ }^{3}$ Max-Planck-Institut für Gravitationsphysik, \\ Am Mühlenberg 1, D-14476 Golm, Germany \\ rendall@aei.mpg.de
}

\begin{abstract}
We prove in the cases of spherical, plane and hyperbolic symmetry a local in time existence theorem and continuation criteria for cosmological solutions of the Einstein-Vlasov-scalar field system, with the sources generated by a distribution function and a scalar field, subject to the Vlasov and wave equations respectively. This system describes the evolution of self-gravitating collisionless matter and scalar waves within the context of general relativity. In the case where the only source is a scalar field it is shown that a global existence result can be deduced from the general theorem.
\end{abstract}

\section{Introduction}

In the mathematical study of general relativity, one of the main problems is to establish the existence and properties of global solutions of the Einstein equations coupled to various matter fields such as collisionless matter described by the Vlasov equation (see [1, 18] for reviews) or a scalar field (see [16] for the cosmological case and [6] and references therein for the asymptotically flat case). An essential tool in an investigation of this type is a local in time existence theorem together with a continuation criterion. In this paper a theorem of this kind is proved for one particular choice of matter model.

In [13 and [14 G. Rein obtained cosmological solutions of the EinsteinVlasov system with surface symmetry written in areal coordinates. In [22] and 
[23, these results were generalized to the case of non-vanishing cosmological constant. In the present paper, we extend the results of [13] on local existence and continuation criteria to the case where the source terms of the Einstein equations are generated by both a distribution function $f$ of particles, which is subject to the Vlasov equation, and a massless scalar field $\phi$, which is subject to the wave equation.

There are several reasons why it is of interest to look at the case of a scalar field. The first is that it is the simplest situation in which wave phenomena can be examined in the context of the Einstein-Vlasov system. In surface symmetry all wave propagation can be eliminated from the Einstein equations by the use of suitable coordinate conditions. This is an analogue of the well-known statement that there are no gravitational waves in spherical symmetry. Mathematically it means that controlling solutions of the Einstein equations can be reduced to controlling solutions of ordinary differential equations in time and in space. The Vlasov equation, being a scalar hyperbolic equation of first order, can also easily be solved in terms of its characteristics. This was the strategy used in [13] and [14. In the presence of a cosmological constant it is possible to follow the same route.

The inclusion of a scalar field introduces waves into the system which cannot be eliminated. Mathematically this means that it introduces a non-trivial hyperbolic equation, the wave equation. This paper is concerned with symmetric situations where there is a symmetry group acting on two-dimensional spacelike orbits. This means that the wave equation reduces to an effective equation in one space dimension. As a consequence part of the strategy used previously can be carried over. That was based on pointwise estimates and not on integral estimates (energy estimates) as is usual in the theory of hyperbolic equations. Pointwise estimates for solutions of wave equations in terms of data can be obtained in one space dimension but not in higher space dimensions (See e.g. the discussion in [10], p. 14.)

Pointwise estimates for hyperbolic equations in one space dimension can be obtained using the method of characteristics. This means that in fact ordinary differential equations appear once again but this time they are integrated not at a constant value of the spatial or time variable but along characteristics. This method will be applied in the following, the characteristics in this case being null curves of the spacetime geometry.

It should be mentioned that there are global existence results in the literature where the Einstein-Vlasov system is considered in a context where hyperbolic equations play an important role. In fact if we relax the assumptions from surface symmetry, where there are three local Killing vectors, to the case where there are only two local spacelike Killing vectors, then hyperbolic equations necessarily occur. Some relevant papers are [17, 4], 24]. In those references no direct local existence proof was given. Instead an indirect argument was used. First a known local existence theorem for the Einstein-Vlasov equation without symmetry was quoted. Then it was shown that coordinates could be introduced which are well-adapted to making use of the symmetry when proceeding to obtain global results. Apart from the methodological interest of having a direct 
local existence proof, the direct proof gives stronger results concerning the differentiability required of the initial data and obtained for the solutions. This is very difficult to control in the indirect method and for this reason the latter has only been applied to the case where everything is of infinite differentiability.

The inclusion of a scalar field can be seen as a step towards certain questions of physical interest. In recent years cosmological models with accelerated expansion have become a very active research topic in response to new astronomical observations [21]. The easiest way to obtain models with accelerated expansion is to introduce a positive cosmological constant, a possibility studied mathematically in [22, [23] and [11]. A more sophisticated way is to introduce a scalar field with potential (see [19], section 4.3., [20, [12]). In the present paper only a linear scalar field is treated but it is likely that the approach developed here will be useful in the nonlinear case. Scalar fields also play a role in theories of gravity generalizing Einstein's theory, such as the Jordan-Brans-Dicke theory. In that case, in contrast to the one considered here, there is a direct coupling between the scalar field and the distribution function. The techniques developed here could serve as a first step towards the study of these more complicated situations, which have hardly been looked at mathematically yet. (See however [2] and [5] where the coupling of the Vlasov equation to a scalar field of the Jordan-Brans-Dicke type in the absence of Einstein gravity is considered.)

We consider a four-dimensional spacetime manifold $M$, with local coordinates $\left(x^{\alpha}\right)=\left(t, x^{i}\right)$ on which $x^{0}=t$ denotes the time and $\left(x^{i}\right)$ the space coordinates. Greek indices always run from 0 to 3 , and Latin ones from 1 to 3 . On $M$, a Lorentzian metric $g$ is given with signature $(-,+,+,+)$. We consider a self-gravitating collisionless gas and restrict ourselves to the case where all particles have the same rest mass, normalized to 1 , and move forward in time. We denote by $\left(p^{\alpha}\right)$ the momenta of the particles. The conservation of the quantity $g_{\alpha \beta} p^{\alpha} p^{\beta}$ requires that the phase space of the particle is the seven-dimensional submanifold

$$
P M=\left\{g_{\alpha \beta} p^{\alpha} p^{\beta}=-1 ; \quad p^{0}>0\right\}
$$

of $T M$ which is coordinatized by $\left(t, x^{i}, p^{i}\right)$. If the coordinates are such that the components $g_{0 i}$ vanish then the component $p^{0}$ is expressed by the other coordinates via

$$
p^{0}=\sqrt{-g^{00}} \sqrt{1+g_{i j} p^{i} p^{j}}
$$

The distribution function of the particles is a non-negative real-valued function denoted by $f$, that is defined on $P M$. In addition we consider a scalar field $\phi$ which is a real-valued function on $M$. The Einstein-Vlasov-scalar field system now reads:

$$
\begin{gathered}
\partial_{t} f+\frac{p^{i}}{p^{0}} \partial_{x^{i}} f-\frac{1}{p^{0}} \Gamma_{\beta \gamma}^{i} p^{\beta} p^{\gamma} \partial_{p^{i}} f=0 \\
\nabla^{\alpha} \nabla_{\alpha} \phi=0 \\
G_{\alpha \beta}=8 \pi T_{\alpha \beta} \\
T_{\alpha \beta}=-\int_{\mathbb{R}^{3}} f p_{\alpha} p_{\beta}|g|^{\frac{1}{2}} \frac{d p^{1} d p^{2} d p^{3}}{p_{0}}+\left(\nabla_{\alpha} \phi \nabla_{\beta} \phi-\frac{1}{2} g_{\alpha \beta} \nabla_{\nu} \phi \nabla^{\nu} \phi\right)
\end{gathered}
$$


where $p_{\alpha}=g_{\alpha \beta} p^{\beta},|g|$ denotes the modulus of determinant of the metric $g_{\alpha \beta}$, $\Gamma_{\alpha \beta}^{\lambda}$ the Christoffel symbols, $G_{\alpha \beta}$ the Einstein tensor, and $T_{\alpha \beta}$ the energymomentum tensor.

Note that since the contribution of $f$ to the energy-momentum tensor is divergence-free [7], the form of the contribution of the scalar field to the energymomentum tensor determines the field equation for $\phi$.

In [15, a definition of spacetimes with spherical, plane and hyperbolic symmetry was given. The spacetime $(M, g)$ is topologically of the form $] 0, \infty\left[\times S^{1} \times\right.$ $S$, where $S$ is a 2 -sphere, a 2 -torus, or a hyperbolic plane, in the case of spherical, plane or hyperbolic symmetry respectively. We now consider a solution of the Einstein-Vlasov-scalar field system where all unknowns are invariant under one of these symmetries and write the Einstein-Vlasov system in areal coordinates. The circumstances under which coordinates of this type exist are discussed in [3. The metric $g$ takes the form

$$
d s^{2}=-e^{2 \mu(t, r)} d t^{2}+e^{2 \lambda(t, r)} d r^{2}+t^{2}\left(d \theta^{2}+\sin _{k}^{2} \theta d \varphi^{2}\right)
$$

where

$$
\sin _{k} \theta= \begin{cases}\sin \theta & \text { for } k=1 \text { (spherical symmetry); } \\ 1 & \text { for } k=0 \text { (plane symmetry); } \\ \sinh \theta & \text { for } k=-1 \text { (hyperbolic symmetry) }\end{cases}
$$

$t>0$ denotes a time-like coordinate, $r \in \mathbb{R}$ and $(\theta, \varphi)$ range in the domains $[0, \pi] \times[0,2 \pi],[0,2 \pi] \times[0,2 \pi],[0, \infty[\times[0,2 \pi]$ respectively, and stand for angular coordinates. The functions $\lambda$ and $\mu$ are periodic in $r$ with period 1 . It has been shown in [13] and [14] that due to the symmetry, $f$ can be written as a function of

$$
t, r, w:=e^{\lambda} p^{1} \text { and } F:=t^{4}\left[\left(p^{2}\right)^{2}+\left(p^{3}\right)^{2}\right],
$$

i.e. $f=f(t, r, w, F)$. In these variables, we have $p^{0}=e^{-\mu} \sqrt{1+w^{2}+F / t^{2}}$. The scalar field is a function of $t$ and $r$ which is periodic in $r$ with period 1 .

We denote by a dot and by a prime the derivatives of the metric components and of the scalar field with respect to $t$ and $r$ respectively. After calculation and using the results of [13, we can write the complete Einstein-Vlasov-scalar field system as follows:

$$
\begin{gathered}
\partial_{t} f+\frac{e^{\mu-\lambda}}{\sqrt{1+w^{2}+F / t^{2}}} \partial_{r} f-\left(\dot{\lambda} w+e^{\mu-\lambda} \mu^{\prime} \sqrt{1+w^{2}+F / t^{2}}\right) \partial_{w} f=0 \\
e^{-2 \mu}(2 t \dot{\lambda}+1)+k=8 \pi t^{2} \rho \\
e^{-2 \mu}(2 t \dot{\mu}-1)-k=8 \pi t^{2} p \\
\mu^{\prime}=-4 \pi t e^{\lambda+\mu} j \\
e^{-2 \lambda}\left(\mu^{\prime \prime}+\mu^{\prime}\left(\mu^{\prime}-\lambda^{\prime}\right)\right)-e^{-2 \mu}\left(\ddot{\lambda}+\left(\dot{\lambda}+\frac{1}{t}\right)(\dot{\lambda}-\dot{\mu})\right)=4 \pi q
\end{gathered}
$$




$$
e^{-2 \lambda} \phi^{\prime \prime}-e^{-2 \mu} \ddot{\phi}-e^{-2 \mu}\left(\dot{\lambda}-\dot{\mu}+\frac{2}{t}\right) \dot{\phi}-e^{-2 \lambda}\left(\lambda^{\prime}-\mu^{\prime}\right) \phi^{\prime}=0
$$

where (1.7) is the wave equation in $\phi$ and :

$$
\begin{gathered}
\begin{aligned}
\rho(t, r)=e^{-2 \mu} T_{00}(t, r)= & \frac{\pi}{t^{2}} \int_{-\infty}^{+\infty} \int_{0}^{+\infty} \sqrt{1+w^{2}+F / t^{2}} f(t, r, w, F) d F d w \\
& +\frac{1}{2}\left(e^{-2 \mu} \dot{\phi}^{2}+e^{-2 \lambda} \phi^{\prime 2}\right)
\end{aligned} \\
\begin{aligned}
p(t, r)=e^{-2 \lambda} T_{11}(t, r)=\frac{\pi}{t^{2}} \int_{-\infty}^{+\infty} \int_{0}^{+\infty} \frac{w^{2}}{\sqrt{1+w^{2}+F / t^{2}}} f(t, r, w, F) d F d w \\
+\frac{1}{2}\left(e^{-2 \mu} \dot{\phi}^{2}+e^{-2 \lambda} \phi^{\prime 2}\right) \\
j(t, r)=-e^{-(\lambda+\mu)} T_{01}(t, r)=\frac{\pi}{t^{2}} \int_{-\infty}^{+\infty} \int_{0}^{+\infty} w f(t, r, w, F) d F d w-e^{-(\lambda+\mu)} \dot{\phi} \phi^{\prime}
\end{aligned} \\
\begin{array}{c}
q(t, r)=\frac{2}{t^{2}} T_{22}(t, r)=\frac{2}{t^{2}} T_{33}(t, r)=\frac{\pi}{t^{4}} \int_{-\infty}^{\infty} \int_{0}^{\infty} \frac{F}{\sqrt{1+w^{2}+F / t^{2}}} f(t, r, w, F) \\
+e^{-2 \mu} \dot{\phi}^{2}-e^{-2 \lambda} \phi^{\prime 2}
\end{array}
\end{gathered}
$$

We are going to study the initial value problem corresponding to this system and prescribe initial data at time $t=1$ :

$$
\begin{aligned}
& f(1, r, w, F)=\stackrel{\circ}{f}(r, w, F), \quad \lambda(1, r)=\stackrel{\circ}{\lambda}(r), \quad \mu(1, r)=\stackrel{\circ}{\mu}(r), \\
& \phi(1, r)=\stackrel{\circ}{\phi}(r), \quad \dot{\phi}(1, r)=\psi(r)
\end{aligned}
$$

The choice $t=1$ is made only for convenience. Analogous results hold in the case that data are prescribed on any hypersurface $t=t_{0}>0$.

The paper is organized as follows. In section 2 , we split the wave equation in $\phi$ into a system of two partial differential equations of first order; we use it to bound the derivatives of $\phi$ by the solutions of the field equations and we introduce an auxiliary system. In section 3 , we solve each equation of the auxiliary system when the other unknowns are fixed. Next, we prove, using the results of [13], that under some constraints on the initial data, the full system is equivalent to the auxiliary system and reduces to a subsystem. Finally we solve the constraint equation on the initial data. In section 4 , we prove local in time existence and uniqueness of solutions in both time directions by iterating the solutions of the auxiliary system and we prove continuation criteria. In section 5 we show that if $f=0$ global existence in the future holds when $k \leq 0$. 


\section{Auxiliary system and preliminary results}

Using characteristic derivatives, we show in this section that the first and second derivatives of $\phi$ can be bounded in terms of $\lambda$ and $\mu$.

Lemma 2.1 Let $D^{+}=e^{-\mu} \partial_{t}+e^{-\lambda} \partial_{r} ; \quad D^{-}=e^{-\mu} \partial_{t}-e^{-\lambda} \partial_{r}$; $X=\dot{\phi} e^{-\mu}-\phi^{\prime} e^{-\lambda} ; Y=\dot{\phi} e^{-\mu}+\phi^{\prime} e^{-\lambda} ;$

$a=\left(-\dot{\lambda}-\frac{1}{t}\right) e^{-\mu}-\mu^{\prime} e^{-\lambda} ; \quad b=-\frac{e^{-\mu}}{t} ; c=\left(-\dot{\lambda}-\frac{1}{t}\right) e^{-\mu}+\mu^{\prime} e^{-\lambda}$ then $X, Y$ are solutions of the system

$$
\begin{aligned}
& D^{+} X=a X+b Y \\
& D^{-} Y=b X+c Y
\end{aligned}
$$

Proof: This results from a simple calculation, since equation (1.7) is satisfied. $\square$

Note that the only derivatives of the metric coefficients which appear in these equations are $\dot{\lambda}$ and $\mu^{\prime}$ and that these are the same ones which appear in the Vlasov equation. This fact plays an important role in the proof of the local existence theorem.

The full system above is overdetermined and we will show that a solution $(f, \lambda, \mu, \phi)$ of the subsystem consisting of equations (1.2), (1.3), (1.4) and (1.7) solves the remaining equations (1.5) and (1.6). Notice that such a solution determines the right hand side of (1.5) which is then a given function say $\tilde{\mu}$. Then, since (1.4) already provides $\mu$, an idea introduced in [13, and that we will follow here, is to replace $\mu^{\prime}$ in (1.2) and (1.7) by an auxiliary function $\tilde{\mu}$, which is not assumed a priori to be a derivative, and prove later that, under certain conditions, $\tilde{\mu}$ is nothing else than $\mu^{\prime}$. We then introduce the following auxiliary system obtained by coupling (1.3)-(1.4) to the equations obtained by replacing $\mu^{\prime}$ by $\tilde{\mu}$ in (1.2), (1.5) and (2.1)-(2.2), i.e :

$$
\begin{gathered}
\partial_{t} f+\frac{e^{\mu-\lambda}}{\sqrt{1+w^{2}+F / t^{2}}} \partial_{r} f-\left(\dot{\lambda} w+e^{\mu-\lambda} \widetilde{\mu} \sqrt{1+w^{2}+F / t^{2}}\right) \partial_{w} f=0 \\
\tilde{\mu}=-4 \pi t e^{\lambda+\mu} j
\end{gathered}
$$

and

$$
\begin{aligned}
& D^{+} X=\tilde{a} X+b Y \\
& D^{-} Y=b X+\tilde{c} Y
\end{aligned}
$$

Where $\mu^{\prime}$ is substituted by $\widetilde{\mu}$ in $a$ and $c$ to obtain $\widetilde{a}$ and $\widetilde{c}$ respectively. We first specify the regularity properties which we require.

Definition 2.2 Let $I \subseteq] 0, \infty[$ be an interval and $(t, r) \in I \times \mathbb{R}$.

a) $f \in C^{1}\left(I \times \mathbb{R}^{2} \times[0, \infty[)\right.$ is regular if $f(t, r+1, w, F)=f(t, r, w, F)$ for $(t, r, w, F) \in I \times \mathbb{R}^{2} \times[0, \infty[, f \geq 0$ and $\operatorname{supp} f(t, r, .,$.$) is compact uniformly in$ $r$ and locally uniformly in $t$. 
b) $\mu \in C^{1}(I \times \mathbb{R})$ is regular, if $\mu^{\prime} \in C^{1}(I \times \mathbb{R})$ and $\mu(t, r+1)=\mu(t, r)$.

c) $\lambda \in C^{1}(I \times \mathbb{R})$ is regular, if $\dot{\lambda} \in C^{1}(I \times \mathbb{R})$ and $\lambda(t, r+1)=\lambda(t, r)$.

d) $\tilde{\mu}\left(\right.$ or $\left.\phi_{1}, \phi_{2}\right) \in C^{1}(I \times \mathbb{R})$ is regular, if $\widetilde{\mu}(t, r+1)=\widetilde{\mu}(t, r)$.

e) $\rho($ or $p, j, q) \in C^{1}(I \times \mathbb{R})$ is regular, if $\rho(t, r+1)=\rho(t, r)$.

f) $\phi \in C^{2}(I \times \mathbb{R})$ is regular, if $\phi(t, r+1)=\phi(t, r)$.

Let us estimate the first and second order derivatives of the scalar field $\phi$, using the characteristic curves of (1.7) and equations (2.5) and (2.6).

Proposition 2.3 Let

$$
\begin{gathered}
K_{0}=2 \sup \left\{|\psi(r)| e^{-\stackrel{\circ}{\mu}(r)}+\left|\stackrel{\circ}{\phi}^{\prime}(r)\right| e^{-\stackrel{\circ}{\lambda}(r)} ; r \in \mathbb{R}\right\} \\
m(t)=\sup \left\{|\dot{\lambda}(t, r)|+\frac{2}{t}+|\tilde{\mu}(t, r)| e^{(\mu-\lambda)(t, r)} ; r \in \mathbb{R}\right\} \\
K(t)=\sup \left\{\left(|X|^{2}+|Y|^{2}\right)^{\frac{1}{2}}(t, r) ; r \in \mathbb{R}\right\}
\end{gathered}
$$

then if $(X, Y)$ is a solution of (2.5) and 2.6) with

$$
X(1)=e^{-\stackrel{\circ}{\mu}(r)} \psi(r)+e^{-\stackrel{\circ}{\lambda}(r) \stackrel{\circ}{\phi}}(r)
$$

and

$$
Y(1)=e^{-\stackrel{\circ}{\mu}(r)} \psi(r)-e^{-\stackrel{\circ}{\lambda}(r) \stackrel{\circ}{\phi}}(r)
$$

then:

1) If $t \in] T, 1], T \geq 0$, we have

$$
K(t) \leq K_{0}+3 \int_{t}^{1} m(s) K(s) d s
$$

2) If $t \geq 1$ the analogous estimate holds with the limits $t$ and 1 exchanged in the integral in (2.9).

Proof: The characteristic curves $\left(t, \gamma_{i}\right), i=1,2$ of the second order partial differential equation (1.7) satisfy the differential equation $\dot{\gamma}_{i}= \pm e^{\lambda-\mu}$. On these characteristic curves, we have from Lemma $2.1, D^{+}=D^{-}=2 e^{-\mu} \frac{d}{d t}$. Then (2.5)-(2.6) become

$$
\left\{\begin{array}{l}
\frac{d}{d t} X\left(t, \gamma_{1}(t)\right)=\frac{1}{2} e^{\mu}(\tilde{a} X+b Y)\left(t, \gamma_{1}(t)\right) \\
\frac{d}{d t} Y\left(t, \gamma_{2}(t)\right)=\frac{1}{2} e^{\mu}(b X+\tilde{c} Y)\left(t, \gamma_{2}(t)\right)
\end{array}\right.
$$

Integrate this system on $[t, 1]$ (or on $[1, t]$ ), take the absolute value in each equation, add the two inequalities and take the supremum of each term to obtain (2.9).

Next, we obtain by direct calculation, using (2.5)-(2.6), the following result: 
Lemma 2.4 Let $D^{+}$and $D^{-}$be defined as in Lemma 2.1 and define $X_{1}=e^{-\lambda} \partial_{r} X, \quad Y_{1}=e^{-\lambda} \partial_{r} Y$;

$b_{1}=\left(-2 \dot{\lambda}-\frac{1}{t}\right) e^{-\mu}-\left(\tilde{\mu}+\mu^{\prime}\right) e^{-\lambda} ; \quad b_{2}=\frac{-e^{-\mu}}{t} ;$

$b_{3}=-\dot{\lambda}^{\prime} e^{-\mu-\lambda}+\left(\lambda^{\prime} \tilde{\mu}-\tilde{\mu} \mu^{\prime}-\tilde{\mu}^{\prime}\right) e^{-2 \lambda} ; \quad b_{4}=\left(-2 \dot{\lambda}-\frac{1}{t}\right) e^{-\mu}+\left(\tilde{\mu}+\mu^{\prime}\right) e^{-\lambda} ;$

$b_{5}=\dot{\lambda}^{\prime} e^{-\mu-\lambda}+\left(\lambda^{\prime} \tilde{\mu}-\tilde{\mu} \mu^{\prime}-\tilde{\mu}^{\prime}\right) e^{-2 \lambda} ;$

If $X$ and $Y$ satisfy (2.5) and (2.6) then $X_{1}$ and $Y_{1}$ satisfy

$$
\begin{gathered}
D^{+} X_{1}=b_{1} X_{1}+b_{2} Y_{1}+b_{3} X \\
D^{-} Y_{1}=b_{2} X_{1}+b_{4} Y_{1}+b_{5} Y
\end{gathered}
$$

Proposition 2.5 Let $K(t)$ be defined as in Proposition 2.3 and set:

$$
\begin{aligned}
A_{0} & =2 \sup \left\{\left[\left(\left|\psi^{\prime}\right|+|\stackrel{\circ}{\mu}||\psi|\right) e^{-\stackrel{\circ}{\mu}}+\left(|\stackrel{\circ}{\phi}|+\left|\stackrel{\circ}{\lambda}^{\prime}\right|\left|\stackrel{\circ}{\phi}^{\prime}\right|\right) e^{-\stackrel{\circ}{\lambda}}\right](r) ; r \in \mathbb{R}\right\} \\
A(t) & =\sup \left\{\left[X_{1}^{2}+Y_{1}^{2}\right](t, r) ; r \in \mathbb{R}\right\} \\
v(t) & =\sup \left\{\frac{2}{t}+4|\dot{\lambda}|+\left(|\tilde{\mu}|+\left|\mu^{\prime}\right|\right) e^{\mu-\lambda}(t, r) ; r \in \mathbb{R}\right\} \\
h(t) & =\sup \left\{\left[\left|\dot{\lambda}^{\prime}\right| e^{-\lambda}+\left(\left|\mu^{\prime}\right||\tilde{\mu}|+\left|\lambda^{\prime}\right||\tilde{\mu}|+\left|\tilde{\mu^{\prime}}\right|\right) e^{\mu-2 \lambda}\right](t, r) ; r \in \mathbb{R}\right\}
\end{aligned}
$$

If in addition to the assumptions of Proposition 2.3 the quantities $X_{1}$ and $Y_{1}$ satisfy 2.10) and (2.11) and agree with $e^{-\lambda} \partial_{r} X$ and $e^{-\lambda} \partial_{r} Y$ respectively for $t=1$ then

1) If $t \in] T, 1], T \geq 0$, we have the estimate

$$
A(t) \leq A_{0}+3 \int_{t}^{1}(v(s) A(s)+h(s) K(s)) d s
$$

2) If $t \geq 1$ the analogous estimate holds with the limits $t$ and 1 exchanged in the integral in (2.12).

Proof: Analogous to the proof of Proposition 2.3, using this time Lemma 2.4. $\square$

Note that the factor $e^{-\lambda}$ in the definition of $X_{1}$ and $Y_{1}$ is very important. Without it the above derivation would not work since the derivative $\lambda^{\prime}$ would occur in $b_{1}$ and $b_{4}$.

\section{The reduced system}

We first solve each equation of the auxiliary system introduced in section 2, when the other unknowns are fixed (in the form to be used later for the iteration). In order to clarify our statements, we introduce the notations $\phi_{1}, \phi_{2}$ in place of $\dot{\phi}$, $\phi^{\prime}$. 
Proposition 3.1 1) Let $\bar{f}, \bar{\lambda}, \bar{\mu}, \tilde{\mu}, \bar{\phi}_{1}, \bar{\phi}_{2}$ be regular for $(t, r) \in I \times \mathbb{R}$, $I \subset] 0, \infty\left[\right.$. Substitute $f, \lambda, \mu, \dot{\phi}, \phi^{\prime}$ respectively by $\bar{f}, \bar{\lambda}, \bar{\mu}, \bar{\phi}_{1}, \bar{\phi}_{2}$ in $\rho$ and $p$ to define $\bar{\rho}$ and $\bar{p}$. Suppose that $1 \in I, \stackrel{\circ}{f} \in C^{1}\left(\mathbb{R}^{2} \times[0, \infty)\right), \stackrel{\circ}{\lambda}, \stackrel{\circ}{\mu} \in C^{1}(\mathbb{R})$ and are periodic of period 1 in $r$. Assume that:

$$
\frac{e^{-2 \stackrel{\circ}{\mu}(r)}+k}{t}-k+\frac{8 \pi}{t} \int_{t}^{1} s^{2} \bar{p}(s, r) d s>0, \quad(t, r) \in I \times \mathbb{R}
$$

then the system

$$
\begin{gathered}
\partial_{t} f+\frac{e^{\bar{\mu}-\bar{\lambda}}}{\sqrt{1+w^{2}+F / t^{2}}} \partial_{r} f-\left(\dot{\bar{\lambda}} w+e^{\bar{\mu}-\bar{\lambda}} \tilde{\mu} \sqrt{1+w^{2}+F / t^{2}}\right) \partial_{w} f=0 \\
e^{-2 \mu}(2 t \dot{\lambda}+1)+k=8 \pi t^{2} \bar{\rho} \\
e^{-2 \mu}(2 t \dot{\mu}-1)-k=8 \pi t^{2} \bar{p}
\end{gathered}
$$

has a unique regular solution $(f, \lambda, \mu)$ on $I \times \mathbb{R}$ with $f(1)=\stackrel{\circ}{f}, \lambda(1)=\stackrel{\circ}{\lambda}$, $\mu(1)=\stackrel{\circ}{\mu}$. This solution is given by

$$
f(t, r, w, F)=\stackrel{\circ}{f}((R, W)(1, t, r, w, F), F)
$$

where $(R, W)$ is the solution of the characteristic system:

$$
\frac{d}{d s}(r, w)=\left(\frac{e^{\bar{\mu}-\bar{\lambda}}}{\sqrt{1+w^{2}+F / t^{2}}},-\dot{\bar{\lambda}} w-e^{\bar{\mu}-\bar{\lambda}} \tilde{\mu} \sqrt{1+w^{2}+F / t^{2}}\right)
$$

satisfying $(R, W)(t, t, r, w, F)=(r, w)$;

$$
\begin{gathered}
e^{-2 \mu(t, r)}=\frac{e^{-2 \stackrel{\circ}{\mu}(r)}+k}{t}-k+\frac{8 \pi}{t} \int_{t}^{1} s^{2} \bar{p}(s, r) d s \\
\dot{\lambda}(t, r)=4 \pi t e^{2 \mu} \bar{\rho}(t, r)-\frac{1+k e^{2 \mu}(t, r)}{2 t} \\
\lambda(t, r)=\stackrel{\circ}{\lambda}(r)-\int_{t}^{1} \dot{\lambda}(s, r) d s
\end{gathered}
$$

If $I=] T, 1]$ (respectively $I=[1, T[$ ) with $T \in[0,1[$ (respectively $T \in] 1, \infty[$ ), then there exists some $T^{*} \in[T, 1]$ (respectively $\left.\left.T^{*} \in\right] 1, T\right]$ ) such that condition (3.1) holds on $\left.] T^{*}, 1\right] \times \mathbb{R}$ (respectively $\left[1, T^{*}\left[\times \mathbb{R}\right.\right.$ ). $T^{*}$ is independent of $\bar{p}$ if $\left.\left.I=\right] T, 1\right]$, whereas it depends on $\bar{p}$ if $I=[1, T[$.

2) Let $\lambda, \mu, \bar{\lambda}, \bar{\mu}$ be regular; let $C, D$ be regular as $\tilde{\mu}$ (see definition 2.2). Set

$$
X=\phi_{1} e^{-\mu}-\phi_{2} e^{-\lambda}, Y=\phi_{1} e^{-\mu}+\phi_{2} e^{-\lambda}
$$


and define the operators $\overline{D^{+}}, \overline{D^{-}}$as $D^{+}, D^{-}$in Lemma 2.1 , with $\lambda, \mu$ substituted respectively by $\bar{\lambda}, \bar{\mu}$. Assume that $\psi \in C^{1}(\mathbb{R}), \stackrel{\circ}{\phi} \in C^{2}(\mathbb{R})$ are periodic of period 1. Then the system

$$
\begin{aligned}
& \bar{D}^{+} X=C \\
& \bar{D}^{-} Y=D
\end{aligned}
$$

has a unique regular solution $\left(\phi_{1}, \phi_{2}\right)$ such that $\left(\phi_{1}, \phi_{2}\right)(1)=(\psi, \stackrel{\circ}{\phi})$.

Proof: 1) the proof of this point is the same as that of Proposition 2.4 in 13 . The only thing added is the existence of $T^{*}$ we now prove and that will replace in the case of local existence, the hypothesis $\stackrel{\circ}{\mu} \leq 0$ if $k=-1$ in 13 .

If $I=] T, 1]$; since $\bar{p} \geq 0$, the left hand side of (3.1) is bounded from below by $h(t, r)=\frac{e^{-2 \mu_{\mu}^{\circ}(r)}+k}{t}-k$. If $k \in\{0,1\}$, we have, since $\frac{1}{t} \geq 1, h(t, r) \geq e^{-2 \stackrel{\circ}{\mu}(r)}>0$ and we can take $T^{*}=T$. If $k=-1$, since $\stackrel{\circ}{\mu}$ is bounded, there exists $\beta>0$, such that $h(1, r)=e^{-2 \mu(r)}>\beta$. By the continuity of $t \mapsto h(t, r)$ at $t=1$, we conclude that:

$$
\left.\left.\left.\left.\exists T^{*} \in\right] T, 1\right] \text { such that } e^{-2 \mu(t, r)} \geq h(t, r)>\frac{\beta}{2}, t \in\right] T^{*}, 1\right] \text { and } \bar{p} \geq 0 .
$$

If $I=[1, T$, define $h(t, r)$ to be all the left hand side of (3.1) and proceed as above in the case $k=-1$, to obtain $T^{*}$ that depends this time on $\bar{p}$.

2) The system (3.11)-(3.12) in $(X, Y)$ is a first order linear hyperbolic system, and the existence of a unique solution with the prescribed data $(X, Y)(1)=$ $\left(e^{-\stackrel{\circ}{\mu}} \psi-e^{-\stackrel{\circ}{\lambda}_{\phi}^{\prime}}, e^{-\stackrel{\circ}{\mu}} \psi+e^{-\stackrel{\circ}{\lambda}_{\phi}^{\prime}}\right)$ is given by a theorem of Friedrichs $[9$. (See also the paper of Douglis [8] where existence of $C^{1}$ solutions of hyperbolic systems in one space dimension was proved for $C^{1}$ initial data in the more general quasilinear case.) We then deduce from the relations (3.10) that define a bijection $(X, Y) \mapsto\left(\phi_{1}, \phi_{2}\right)$, the existence of a unique regular solution $\left(\phi_{1}, \phi_{2}\right)$ of (3.11)(3.12) such that $\left(\phi_{1}, \phi_{2}\right)(1)=(\psi, \stackrel{\circ}{\phi})$. This completes the proof of Proposition 3.1.

In fact, since the equations for $X$ and $Y$ are decoupled, it is not necessary to use existence results for hyperbolic systems in the above proof; it suffices to solve a parameter-dependent ordinary differential equation. The above procedure has the advantage that it can easily be generalized to problems where the corresponding equations are coupled as they are, for instance, in the case of a nonlinear scalar field.

Now we show that the solution of the subsystem consisting of equations (1.2), (1.3), (1.4) and (1.7) also satisfies equations (1.5) and (1.6), and that the auxiliary system is equivalent to the full system.

Proposition 3.2 1) Let $(f, \lambda, \mu, \phi)$ be a regular solution of (1.2), (1.3), (1.4) and (1.7) on some time interval $I \in] 0, \infty[$ with $1 \in I$, and let the initial data 
satisfy (1.5) for $t=1$ with $\stackrel{\circ}{\phi}, \psi \in C^{1}(\mathbb{R})$, in particular $\stackrel{\circ}{\mu} \in C^{2}(\mathbb{R})$. Then (1.5) and (1.6) hold for all $t \in I$, in particular $\mu \in C^{2}(I \times \mathbb{R})$.

2) Let $\left(f, \lambda, \mu, \tilde{\mu}, \phi_{1}, \phi_{2}\right)$ be a regular solution of equations (2.3), (1.3), 1.4), 2.4), 2.5) and (2.6) with the initial data $\left(f, \lambda, \mu, \phi_{1}, \phi_{2}\right)(1)=(\stackrel{\circ}{f}, \stackrel{\circ}{\lambda}, \stackrel{\circ}{\mu}, \psi, \stackrel{\circ}{\phi})$ that are as in proposition [3.1 and satisfy (1.5) for $t=1$. Then there exists a unique regular function $\phi$ satisfying $\phi(1)=\stackrel{\circ}{\phi}, \dot{\phi}(1)=\psi$ such that $(f, \lambda, \mu, \phi)$ solves the full system [1.2)-(1.11). The function $\phi$ is given by: $\phi(t, r)=\stackrel{\circ}{\phi}(r)+$ $\int_{1}^{t} \phi_{1}(s, r) d s$.

Proof: 1) Firstly, we prove that (1.5) holds. From equations (1.9), (1.2), (1.7) and integration by parts, it follows that

$$
\begin{array}{r}
\int_{1}^{t} p^{\prime}(s, r) s^{2} d s=\pi \int_{1}^{t} \int_{-\infty}^{\infty} \int_{0}^{\infty} \frac{w^{2}}{\sqrt{1+w^{2}+F / s^{2}}} \partial_{r} f(s, r, w, F) d F d w d s \\
+\int_{1}^{t}\left(-\mu^{\prime} \dot{\phi}^{2} e^{-2 \mu}-\lambda^{\prime} \phi^{\prime 2} e^{-2 \lambda}+\dot{\phi}^{\prime} \phi^{\prime} e^{-2 \mu}+\phi^{\prime} \phi^{\prime \prime} e^{-2 \lambda}\right) s^{2} d s \\
=\left[-e^{\lambda-\mu} j(s, r) s^{2}\right]_{s=1}^{s=t}-\int_{1}^{t}(\dot{\lambda}+\dot{\mu}) e^{\lambda-\mu} j(s, r) s^{2} d s-\int_{1}^{t} \mu^{\prime}(\rho+p)(s, r) s^{2} d s
\end{array}
$$

From equation (1.4), we obtain

$$
e^{-2 \mu(t, r)}=\frac{e^{-2 \stackrel{\circ}{\mu}(r)}+k}{t}-k+\frac{8 \pi}{t} \int_{t}^{1} s^{2} p(s, r) d s
$$

and differentiating this with respect to $r$, yields:

$$
t \mu^{\prime} e^{-2 \mu}=\stackrel{\circ}{\mu} e^{-2 \stackrel{\circ}{\mu}}+4 \pi \int_{1}^{t} s^{2} p^{\prime}(s, r) d s
$$

Substituting for the last integral by (3.14), using (1.3) and (1.4) to replace $\dot{\lambda}+\dot{\mu}$ and assuming the fact that the constraint equation (1.5) holds for $t=1$, relation (3.15) implies

$$
-t e^{2 \mu}\left(\mu^{\prime}+4 \pi e^{\lambda+\mu} j t\right)=4 \pi \int_{1}^{t}(\rho+p) s^{2}\left(\mu^{\prime}+4 \pi e^{\lambda+\mu} j s\right) d s
$$

and since the left hand side is zero at $t=1$, we obtain

$$
\mu^{\prime}+4 \pi t e^{\lambda+\mu} j=0
$$

on $I$, i.e (1.5) holds for all $t \in I$. In particular this relation shows that $\mu$ is $C^{2}$ with respect to $r$ with

$$
\mu^{\prime \prime}=\left(\lambda^{\prime}+\mu^{\prime}\right) \mu^{\prime}-4 \pi t e^{\lambda+\mu} j^{\prime} .
$$


Now we prove that equation (1.6) holds. From (1.10), (1.2), (1.7) and integration by parts we obtain the identity

$$
\begin{aligned}
j^{\prime}(t, r) & =\frac{\pi}{t^{2}} e^{\lambda-\mu} \int_{-\infty}^{\infty} \int_{0}^{\infty}\left[-\sqrt{1+w^{2}+F / t^{2}} \partial_{t} f\right. \\
& \left.+\left(\dot{\lambda} w \sqrt{1+w^{2}+F / t^{2}}+e^{\mu-\lambda} \mu^{\prime}\left(1+w^{2}+F / t^{2}\right)\right) \partial_{w} f\right] d F d w \\
& +2 \mu^{\prime} e^{-(\lambda+\mu)} \dot{\phi} \phi^{\prime}-e^{-(\lambda+\mu)} \dot{\phi}^{\prime} \phi^{\prime}-e^{\lambda-3 \mu}\left[\dot{\phi} \ddot{\phi}+\left(\dot{\lambda}-\dot{\mu}+\frac{2}{t}\right) \dot{\phi}^{2}\right] \\
& =-\frac{\pi}{t^{2}} e^{\lambda-\mu} \int_{-\infty}^{\infty} \int_{0}^{\infty} \sqrt{1+w^{2}+F / t^{2}} \partial_{t} f d F d w-\dot{\lambda} e^{\lambda-\mu}\left(\rho+p-\dot{\phi}^{2} e^{-2 \mu}\right. \\
& \left.-\phi^{\prime 2} e^{-2 \lambda}\right)-2 \mu^{\prime} j-e^{-(\lambda+\mu)} \dot{\phi}^{\prime} \phi^{\prime}-e^{-3 \mu+\lambda}\left(\dot{\phi} \ddot{\phi}-\left(\dot{\lambda}-\dot{\mu}+\frac{2}{t}\right) \dot{\phi}^{2}\right) .
\end{aligned}
$$

Since by (1.8)

$$
\begin{aligned}
\dot{\rho}(t, r) & =-2 \frac{\pi}{t^{3}} \int_{-\infty}^{\infty} \int_{0}^{\infty} \sqrt{1+w^{2}+F / t^{2}} f d F d w \\
& +\frac{\pi}{t^{2}} \int_{-\infty}^{\infty} \int_{0}^{\infty} \sqrt{1+w^{2}+F / t^{2}} \partial_{t} f d F d w \\
& +\frac{\pi}{t^{2}} \int_{-\infty}^{\infty} \int_{0}^{\infty}\left(-\frac{F}{t^{3}}\right) \frac{f}{\sqrt{1+w^{2}+F / t^{2}}} d F d w \\
& +e^{-2 \mu}\left(-\dot{\mu} \dot{\phi}^{2}+\dot{\phi} \ddot{\phi}\right)+e^{-2 \lambda}\left(-\dot{\lambda} \phi^{\prime 2}+\dot{\phi}^{\prime} \phi^{\prime}\right) \\
& =\frac{-2 \rho}{t}-\frac{q}{t}+\frac{2 e^{-2 \mu}}{t} \dot{\phi}^{2}+e^{-2 \mu}\left(-\dot{\mu} \dot{\phi}^{2}+\dot{\phi} \ddot{\phi}\right)+e^{-2 \lambda}\left(-\dot{\lambda} \phi^{\prime 2}+\dot{\phi}^{\prime} \phi^{\prime}\right) \\
& +\frac{\pi}{t^{2}} \int_{-\infty}^{\infty} \int_{0}^{\infty} \sqrt{1+w^{2}+F / t^{2}} \partial_{t} f d F d w ;
\end{aligned}
$$

From (1.3) we obtain

$$
\dot{\lambda}(t, r)=4 \pi t e^{2 \mu} \rho(t, r)-\frac{1+k e^{2 \mu}(t, r)}{2 t}
$$

and differentiating with respect to $t$ yields:

$$
\begin{aligned}
\ddot{\lambda} & =4 \pi e^{2 \mu} \rho+8 \pi t \dot{\mu} e^{2 \mu} \rho+\frac{1+k e^{2 \mu}}{2 t^{2}}+4 \pi t e^{2 \mu} \dot{\rho}-\frac{k \dot{\mu} e^{2 \mu}}{t} \\
& =\frac{\dot{\mu}-\dot{\lambda}}{t}-4 \pi q e^{2 \mu}+2 \dot{\lambda} \dot{\mu}+8 \pi \dot{\phi}^{2}+4 \pi t\left(-\dot{\mu} \dot{\phi}^{2}+\dot{\phi} \ddot{\phi}\right)+4 \pi t e^{2 \mu-2 \lambda}\left(-\dot{\lambda}{\phi^{\prime}}^{2}+\dot{\phi}^{\prime} \phi^{\prime}\right) \\
& +4 \frac{\pi^{2}}{t} e^{2 \mu} \int_{-\infty}^{\infty} \int_{0}^{\infty} \sqrt{1+w^{2}+F / t^{2}} \partial_{t} f d F d w
\end{aligned}
$$

Combining all these relations gives the remaining field equation (1.6).

2) subtract the two equations (2.5)-(2.6) to obtain

$$
\dot{\phi}_{2}-\phi_{1}^{\prime}=\left(\tilde{\mu}-\mu^{\prime}\right) \phi_{1}
$$


Let us first prove that $\mu^{\prime}=\tilde{\mu}$. Consider equation 3.15. and write $p=p_{1}+p_{2}$ where $p_{1}$ and $p_{2}$ are the contributions to $p$ made by $f$ and $\phi$ respectively. We obtain from [13] that studies the case $\phi=0$, and in which $p$ corresponds to $p_{1}$ here, that we have, using definitions (1.8), (1.9) and (1.10) of $\rho, p, j$ :

$$
\begin{aligned}
\int_{1}^{t} s^{2} p_{1}^{\prime}(s, r) d s & =-\int_{1}^{t}(\dot{\lambda}+\dot{\mu}) e^{\lambda-\mu} s^{2}\left(j+e^{-\lambda-\mu} \phi_{1} \phi_{2}\right) d s-\left[e^{\lambda-\mu} s^{2}\left(j+e^{-\lambda-\mu} \phi_{1} \phi_{2}\right)\right]_{1}^{t} \\
& -\int_{1}^{t} \tilde{\mu}\left[(\rho+p)-\left(e^{-2 \mu} \phi_{1}^{2}+e^{-2 \lambda} \phi_{2}^{2}\right)\right] s^{2} d s
\end{aligned}
$$

Now we obtain, using integration by parts and (3.16) that:

$$
\int_{1}^{t} s^{2} p_{2}^{\prime}(s, r) d s=\left[s^{2} e^{-2 \mu} \phi_{1} \phi_{2}\right]_{1}^{t}+\int_{1}^{t}\left[(\dot{\lambda}+\dot{\mu}) \phi_{1} \phi_{2} e^{-2 \mu}-\tilde{\mu}\left(\phi_{1}^{2} e^{-2 \mu}+\phi_{2}^{2} e^{-2 \lambda}\right)\right] s^{2} d s
$$

Adding (1.3) and (1.4) yields

$$
\dot{\lambda}+\dot{\mu}=4 \pi t e^{2 \mu}(\rho+p)
$$

Then, if we use (3.17) and the definition (2.4) of $\tilde{\mu}$, we obtain from :

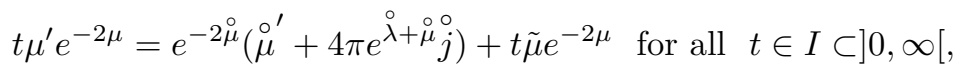

Hence, if (1.5) holds for $t=1$, then, $t \mu^{\prime} e^{-2 \mu}=t \tilde{\mu} e^{-2 \mu}$ and $\mu^{\prime}=\tilde{\mu}$.

Now we prove the existence of $\phi$. Define $\phi$ by : $\phi(t, r)=\stackrel{\circ}{\phi}(r)+\int_{1}^{t} \phi_{1}(s, r) d s$. Then $\phi(1)=\stackrel{\circ}{\phi}, \dot{\phi}(1)=\phi_{1}(1)=\psi$ and $\dot{\phi}=\phi_{1}$; Now (3.16) implies, since $\mu^{\prime}=\tilde{\mu}$, that $\dot{\phi}_{2}=\phi_{1}^{\prime}$, hence the relation $\phi_{2}(1)=\phi$ implies $\phi^{\prime}=\phi_{2}$.

The relation $\mu^{\prime}=\tilde{\mu}$ also implies that the systems (2.1)-(2.2) and (2.5)-(2.6) are identical. Then a direct calculation, using the fact that $\left(\phi_{1}, \phi_{2}\right)$ satisfies the system (2.1)-(2.2) shows that $\phi$ satisfies (1.7).

We conclude this section with a proposition dealing with the solvability of the constraint equation (1.5) for $t=1$. Let $\tilde{\psi}=e^{-\mu} \psi$.

Proposition 3.3 Given a function $\stackrel{\circ}{\lambda}(r)$, a non-negative function $\bar{f}(r, w, F)$ and functions $\stackrel{\circ}{\phi}(r)$ and $\tilde{\psi}(r)$, all periodic in $r$ and regular, there exists a function $\stackrel{\circ}{\mu}(r)$, periodic in $r$ and regular, such that the constraint equation

$$
\stackrel{\circ}{\mu}=-4 \pi e^{\stackrel{\circ}{\lambda}+\stackrel{\circ}{\mu}} j
$$

holds for a non-negative function $\stackrel{\circ}{f}$. It can be assumed that $\stackrel{\circ}{f}=\bar{f}+a \Phi$, where $\Phi(r, w, F)$ is a fixed function, independent of the particular choice of input data, and $a$ is a suitable constant. 
Proof: This can be proved just as in [22, with $\Phi$ chosen as in that reference.

This result shows that it is possible to produce a plentiful supply of initial data. It cannot be applied to produce data with $f=0$. A way of doing that is to adjust $\tilde{\psi}$ instead of adjusting $f$.

\section{Local existence and continuation of solutions}

In this section we prove using an iteration the local existence and uniqueness of solutions of the Einstein-Vlasov-scalar field system together with continuation criteria. Let us first use the solution $\left(f, \lambda, \mu, \tilde{\mu}, \phi_{1}, \phi_{2}\right)$ of the auxiliary system consisting of the equations (2.3), (1.3), (1.4), (2.4), (2.5) and (2.6), to construct a sequence of iterative solutions as follows. Define $\underset{\tilde{\mu}}{\tilde{\rho}}:=\stackrel{\circ}{\prime} \mu^{\prime}, \lambda_{0}(t, r):=\stackrel{\circ}{\lambda}(r)$, $\mu_{0}(t, r):=\stackrel{\circ}{\mu}(r), \tilde{\mu}_{0}(t, r):=\stackrel{\circ}{\tilde{\mu}}(r), g_{0}(t, r)=\psi(r), h_{0}(t, r)=\stackrel{\circ}{\phi}$ for $\left.\left.t \in\right] 0,1\right]$, $r \in \mathbb{R}$. If $\lambda_{n-1}, \mu_{n-1}, \tilde{\mu}_{n-1}$ are already defined and regular on $\left.] T^{*}, 1\right] \times \mathbb{R}$ then let

$G_{n-1}(t, r, w, F):=\left(\frac{w e^{\mu_{n-1}-\lambda_{n-1}}}{\sqrt{1+w^{2}+F / t^{2}}},-\dot{\lambda}_{n-1} w-e^{\mu_{n-1}-\lambda_{n-1}} \tilde{\mu}_{n-1} \sqrt{1+w^{2}+F / t^{2}}\right)$

and denote by $\left(R_{n}, W_{n}\right)(s, t, r, w, F)$ the solution of the characteristic system

$$
\frac{d}{d s}(R, W)=G_{n-1}(s, R, W, F)
$$

with initial data

$$
\left.\left.\left(R_{n}, W_{n}\right)(t, t, r, w, F)=(r, w) ; \quad(t, r, w, F) \in\right] 0,1\right] \times \mathbb{R}^{2} \times[0, \infty[;
$$

note that $F$ is constant along characteristics. Define

$$
f_{n}(t, r, w, F):=\stackrel{\circ}{f}\left(\left(R_{n}, W_{n}\right)(1, t, r, w, F), F\right),
$$

that is, $f_{n}$ is the solution of

$\partial_{t} f_{n}+\frac{w e^{\mu_{n-1}-\lambda_{n-1}}}{\sqrt{1+w^{2}+F / t^{2}}} \partial_{r} f_{n}-\left(\dot{\lambda}_{n-1} w+e^{\mu_{n-1}-\lambda_{n-1}} \tilde{\mu}_{n-1} \sqrt{1+w^{2}+F / t^{2}}\right) \partial_{w} f_{n}=0$

with $f_{n}(1)=\stackrel{\circ}{f}$. Define $\rho_{n}, p_{n}, j_{n}, q_{n}$ by the formulas (1.8), (1.9), (1.10) and (1.11) with $f, \lambda, \mu, \dot{\phi}, \phi^{\prime}$ respectively replaced by $f_{n}, \lambda_{n-1}, \mu_{n-1}, g_{n-1}, h_{n-1}$, $(n \geq 1)$. Using Proposition 3.1,1), define $\mu_{n}$ and $\lambda_{n}$ to be the solutions of

$$
e^{-2 \mu_{n}(t, r)}=\frac{e^{-2 \stackrel{\circ}{\mu}(r)}+k}{t}-k+\frac{8 \pi}{t} \int_{t}^{1} s^{2} p_{n}(s, r) d s
$$




$$
\begin{gathered}
\dot{\lambda}_{n}(t, r)=4 \pi t e^{2 \mu_{n}(t, r)} \rho_{n}(t, r)-\frac{1+k e^{2 \mu_{n}}}{2 t} \\
\lambda_{n}(t, r)=\stackrel{\circ}{\lambda}(r)-\int_{t}^{1} \dot{\lambda}_{n}(s, r) d s
\end{gathered}
$$

and set

$$
\tilde{\mu}_{n}(t, r)=-4 \pi t e^{\left(\mu_{n}+\lambda_{n}\right)(t, r)} j_{n}(t, r)
$$

Notice that, by Proposition 3.1, the right hand side of (4.4) is positive on $\left.] T^{*}, 1\right]$, $\forall n$. Now define $g_{n}$ and $h_{n}$ using Proposition 3.1,2) to satisfy the conditions that the quantities

$$
X_{n}=e^{-\mu_{n}} g_{n}-e^{-\lambda_{n}} h_{n}, \quad Y_{n}=e^{-\mu_{n}} g_{n}+e^{-\lambda_{n}} h_{n}
$$

are solutions of the system

$$
\begin{aligned}
& D_{n-1}^{+} X_{n}=a_{n-1} X_{n-1}+b_{n-1} Y_{n-1} \\
& D_{n-1}^{-} Y_{n}=b_{n-1} X_{n-1}+c_{n-1} Y_{n-1}
\end{aligned}
$$

where $D_{n-1}^{+}, D_{n-1}^{-}, a_{n-1}, b_{n-1}$ and $c_{n-1}$ are defined in the same way as $D^{+}$, $D^{-}, \tilde{a}, b, \tilde{c}$ (see Lemma 2.1), with $\mu, \lambda, \dot{\phi}, \phi^{\prime}, \tilde{a}, b, \tilde{c}$ substituted respectively by $\mu_{n-1}, \lambda_{n-1}, g_{n-1}, h_{n-1}, a_{n-1}, b_{n-1}, c_{n-1}$. Now $K_{0}$ and $A_{0}$ being defined in Propositions 2.3 and 2.5, we introduce the following quantities that are similar to those defined in those propositions:

$$
\begin{gathered}
K_{n}(t)=\sup \left\{\left(g_{n}^{2} e^{-2 \mu_{n}}+h_{n}^{2} e^{-2 \lambda_{n}}\right)^{\frac{1}{2}}(t, r) ; r \in \mathbb{R}\right\} \\
\left\{\begin{array}{c}
A_{n}(t)=\sup \left\{e^{-2 \lambda_{n}}\left[\left(g_{n}^{\prime}-\mu_{n}^{\prime} g_{n}\right)^{2} e^{-2 \mu_{n}}+\left(h_{n}^{\prime}-\lambda_{n}^{\prime} h_{n}\right)^{2} e^{-2 \lambda_{n}}\right](t, r) ; r \in \mathbb{R}\right\} \\
m_{n-1}(t)=\sup \left\{\frac{2}{t}+\left(\left|\dot{\lambda}_{n-1}\right|+\left|\tilde{\mu}_{n-1}\right| e^{\mu_{n-1}-\lambda_{n-1}}\right)(t, r) ; r \in \mathbb{R}\right\} \\
v_{n-1}(t)=\sup \left\{\frac{2}{t}+4\left|\dot{\lambda}_{n-1}\right|+\left(\left|\tilde{\mu}_{n-1}\right|+\left|\mu_{n-1}^{\prime}\right|\right) e^{\mu_{n-1}-\lambda_{n-1}}(t, r) ; r \in \mathbb{R}\right\} \\
\beta_{n-1}(t)=\sup \left\{\left[\left|\dot{\lambda}_{n-1}^{\prime}\right| e^{-\lambda_{n-1}}+\left(\left|\mu_{n-1}^{\prime}\right|\left|\tilde{\mu}_{n-1}\right|+\left|\lambda_{n-1}^{\prime} \| \tilde{\mu}_{n-1}\right|\right.\right.\right. \\
\left.\left.\left.+\left|\tilde{\mu}_{n-1}^{\prime}\right|\right) e^{\mu_{n-1}-2 \lambda_{n-1}}\right](t, r) ; r \in \mathbb{R}\right\}
\end{array}\right.
\end{gathered}
$$

Now we proceed for (4.8)-(4.9) the same way as we did for (2.5)-(2.6) to establish the inequality (2.9) and we obtain the following analogous inequality:

$$
K_{n}(t) \leq K_{0}+3 \int_{t}^{1} m_{n-1}(s) K_{n-1}(s) d s
$$

We can use (4.8)-(4.9) to establish a system for $\left(e^{-\lambda_{n-1}} \partial_{r} X_{n}, e^{-\lambda_{n-1}} \partial_{r} Y_{n}\right)$ analogous to (2.10)-(2.11) from which we deduce the following inequality which is analogous to (2.12)

$$
A_{n}(t) \leq A_{0}+3 \int_{t}^{1}\left(v_{n-1}(s) A_{n-1}(s)+\beta_{n-1}(s) K_{n-1}(s)\right) d s
$$


Throughout the paper, $\|$. $\|$ denotes the $L^{\infty}$-norm on the function space in question; we use the fact that by (4.2), $\left\|f_{n}(t)\right\|=\|\stackrel{\circ}{f}\|$ for $n \in \mathbb{N}$ and $\left.t \in] T^{*}, 1\right]$. The numerical constant $C$ may change from line to line and does not depend on $n$ or $t$ or the initial data. In order to prove the local existence theorem, we prove respectively in the next two propositions:

- a uniform bound on the momenta in the support of distribution functions $f_{n}$, and a uniform bound of the first derivatives with respect to $r$ of the functions $f_{n}, \lambda_{n}, \mu_{n}, g_{n}, h_{n}$

- the convergence of the iterates.

Proposition 4.1 We take $\stackrel{\circ}{f}$ as in proposition 3.1 and such that

$$
\operatorname{supp} \stackrel{\circ}{f} \subset\left[0, W_{0}\right] \times\left[0, F_{0}\right], W_{0}>0, F_{0}>0 .
$$

then there exist nonnegative constants $T_{1}, T_{2}$ such that the quantities

$$
\begin{gathered}
Q_{n}(t)=\sup \left\{|w| ; \quad(r, w, F) \in \operatorname{supp} f_{n}(t)\right\} \text { for all } t \in\left[T_{1}, 1\right] . \\
B_{n}(t)=\sup \left\{\left\|\partial_{r} f_{n}(s)\right\|+A_{n-1}(s) ; t \leq s \leq 1\right\} \text { for all } t \in\left[T_{2}, 1\right] .
\end{gathered}
$$

and $K_{n}(t)$ are uniformly bounded in $n$.

Proof: Firstly we bound $Q_{n}(t)$ and $K_{n}(t)$. On $\operatorname{supp} f_{n}(t)$, we have

$$
\sqrt{1+w^{2}+F / t^{2}} \leq \sqrt{1+Q_{n}^{2}+F_{0} / t^{2}} \leq \frac{1}{t}\left(1+F_{0}\right)\left(1+Q_{n}(t)\right)
$$

and thus

$$
\left\|\rho_{n}(t)\right\|,\left\|p_{n}(t)\right\|,\left\|j_{n}(t)\right\| \leq \frac{C}{t^{3}}\left(1+F_{0}\right)^{2}\left(1+Q_{n}(t)\right)^{2}\|\stackrel{\circ}{f}\|+\left(K_{n-1}(t)\right)^{2}
$$

From (3.13), we have, setting $C_{0}=\frac{\beta}{2}$,

$$
e^{-2 \mu_{n}}(t, r) \geq \frac{C_{0}}{t}
$$

Hence, proceeding as in step 1 of the proof of theorem 3.1 in 13 and using the expression for $j_{n}$, we have :

$$
Q_{n+1}(t) \leq W_{0}+C_{1} \int_{t}^{1} \frac{1}{s}\left(1+Q_{n}(s)\right)^{2}\left(1+K_{n-1}(s)\right)^{2}\left(1+Q_{n+1}(s)\right) d s
$$

with $C_{1}=\frac{C}{C_{0}}\left[\left(1+F_{0}\right)^{2}(1+\|\stackrel{\circ}{f}\|)\right.$. Next we have, using (4.5)-(4.7) and (4.15)

$$
\left|\dot{\lambda_{n}}(s, r)\right|+\left|\left(\tilde{\mu}_{n} e^{\mu_{n}-\lambda_{n}}\right)(s, r)\right| \leq C_{1} \frac{\left(1+Q_{n}(s)\right)^{2}}{s}\left(1+K_{n-1}(s)\right)^{2}
$$

we then deduce from (4.11) that

$$
K_{n+1}(t) \leq K_{0}+C_{1} \int_{t}^{1} \frac{\left(1+Q_{n}(s)\right)^{2}}{s}\left(1+K_{n-1}(s)\right)^{2} K_{n}(s) d s
$$


Add (4.16) and (4.18) to obtain

$$
Q_{n+1}(t)+K_{n+1}(t) \leq W_{0}+K_{0}+C_{1} \int_{t}^{1} \frac{1}{s}\left(1+Q_{n}(s)\right)^{2}\left(1+K_{n-1}(s)\right)^{2}\left(1+Q_{n+1}(s)+K_{n}(s)\right) d s
$$

Now define $H_{n}(t):=\sup \left\{Q_{m}(t)+K_{m}(t) ; m \leq n\right\} .\left(H_{n}\right)_{n \in \mathbb{N}}$ is an increasing sequence. Then, use (4.19) and the inequalities obtained by replacing in (4.19), $n$ by any $m \leq n$, to obtain :

$$
H_{n+1}(t) \leq W_{0}+K_{0}+C_{1} \int_{t}^{1} \frac{1}{s}\left(1+H_{n+1}(s)\right)^{5} d s
$$

Let $z_{1}$ be the left maximal solution of the equation

$$
z_{1}(t)=W_{0}+K_{0}+C_{1} \int_{t}^{1} \frac{1}{s}\left(1+z_{1}(s)\right)^{5} d s
$$

which exists on some interval $\left.] T_{1}, 1\right]$ with $T_{1} \in\left[T^{*}, 1[\right.$. By comparing the solution of the integral inequality with that of the corresponding integral equation it follows that

$$
\left.H_{n+1}(t) \leq z_{1}(t), t \in\right] T_{1}, 1[, n \in \mathbb{N} .
$$

Since $Q_{n}(t)+K_{n}(t) \leq H_{n+1}(t)$, we obtain

$$
\left.K_{n}(t), Q_{n}(t) \leq z_{1}(t), t \in\right] T_{1}, 1[, n \in \mathbb{N} .
$$

And all the quantities which were estimated against $Q_{n}$ and $K_{n}$ in the above argument are bounded by certain powers of $z_{1}$ on $\left.] T_{1}, 1\right]$. Namely there exists a continuous function $C_{2}(t)$ which depends only on $z_{1}$ as an increasing function, such that

$$
\left\{\begin{array}{c}
\left\|\mu_{n}(t)\right\|,\left\|\lambda_{n}(t)\right\|,\left\|\dot{\lambda}_{n}(t)\right\|,\left\|\rho_{n}(t)\right\|,\left\|p_{n}(t)\right\|, \\
\left\|j_{n}(t)\right\|,\left\|\tilde{\mu}_{n} e^{\mu_{n}-\lambda_{n}}\right\|,\left\|g_{n}(t)\right\|,\left\|h_{n}(t)\right\| \leq C_{2}(t)
\end{array}\right.
$$

Now we bound $B_{n}(t)$. Following step 2 of the proof of theorem 3.1 in [13], we have, using (4.20), the estimates

$\left\|\rho_{n}^{\prime}(t)\right\|,\left\|p_{n}^{\prime}(t)\right\|,\left\|j_{n}^{\prime}(t)\right\|,\left\|\mu_{n}^{\prime}(t)\right\|,\left\|\dot{\lambda}_{n}^{\prime}(t)\right\|,\left\|\lambda_{n}^{\prime}(t)\right\| \leq C_{2}(t)\left(C_{3}+B_{n}(t)\right)$

$$
\left\|\tilde{\mu}_{n}^{\prime} e^{\mu_{n}-\lambda_{n}}\right\| \leq C_{2}(t)\left(C_{3}+B_{n}(t)\right)
$$

$$
\left\|\partial_{r} f_{n+1}(t)\right\| \leq\left\|\partial_{(r, w)} \stackrel{\circ}{f}\right\| \exp \int_{t}^{1} C_{2}(s)\left(C_{3}+B_{n}(s)\right) d s
$$

with $C_{3}=\|\stackrel{\circ}{\lambda}\|+\left\|\stackrel{\circ}{\mu}^{\prime} e^{-2 \stackrel{\circ}{\mu}}\right\|+1$. We use (4.20), (4.21), (4.22) and relation (4.12) to obtain

$$
A_{n+1}(t) \leq A_{0}+\int_{t}^{1} C_{2}(s)\left(C_{3}+B_{n}(s)\right) A_{n}(s) d s
$$


Let $D_{n}(t):=\sup \left\{A_{m}(t) \mid m \leq n\right\}$ and $E_{n}(t):=\sup \left\{B_{m}(t) \mid m \leq n\right\} .\left\{D_{n}\right\}$ and $\left\{E_{n}\right\}$ are increasing sequences. Therefore

$$
A_{n+1}(t) \leq A_{0}+\int_{t}^{1} C_{2}(s)\left(C_{3}+E_{n}(s)\right) D_{n+1}(s) d s
$$

then we deduce by replacing $n$ by any $m \leq n$ in (4.24)

$$
D_{n+1}(t) \leq A_{0}+\int_{1}^{t} C_{2}(s)\left(C_{3}+E_{n}(s)\right) D_{n+1}(s) d s
$$

Which gives:

$$
D_{n+1}(t) \leq 2 A_{0} \exp \int_{t}^{1} C_{2}(s)\left(C_{3}+E_{n}(s)\right) d s
$$

Now add (4.23)-(4.25) to obtain

$$
B_{n+1}(t) \leq\left(2 A_{0}+\left\|\partial_{r, w} f\right\|\right) \exp \int_{t}^{1} C_{2}(s)\left(C_{3}+E_{n}(s)\right) d s
$$

and deduce by replacing $n$ by every $m \leq n$ that:

$$
E_{n+1}(t) \leq C_{4} \exp \int_{t}^{1} C_{2}(s)\left(C_{3}+E_{n+1}(s)\right) d s
$$

where $C_{4}=2 A_{0}+\left\|\partial_{(r, w)} \stackrel{\circ}{f}\right\|$. Let $z_{2}$ be the left maximal solution of

$$
z_{2}(t)=C_{4} \exp \int_{t}^{1} C_{2}(s)\left(C_{3}+z_{2}(s)\right) d s
$$

i.e

$$
\dot{z}_{2}(t)=-C_{2}(t)\left(C_{3}+z_{2}(t)\right) z_{2}(t), \quad z_{2}(1)=C_{4} ;
$$

which exists on an interval $\left.\left.\left.] T_{2}, 1\right] \subset\right] T_{1}, 1\right]$. Then we have

$$
\left.\left.E_{n+1}(t) \leq z_{2}(t), t \in\right] T_{2}, 1\right], n \in \mathbb{N}
$$

and so

$$
\left.\left.A_{n}(t), B_{n}(t) \leq z_{2}(t), t \in\right] T_{2}, 1\right], n \in \mathbb{N}
$$

and all the quantities estimated against $B_{n}$ above can be bounded in terms of $z_{2}$ on $\left.] T_{2}, 1\right]$, uniformly in $n$. $\square$

Remark 4.2 The sequences $\lambda_{n}, \mu_{n}, f_{n}, \tilde{\mu}_{n} e^{\mu_{n}-\lambda_{n}}, \rho_{n}, p_{n}, j_{n}, g_{n}, h_{n}, \lambda_{n}^{\prime}, \mu_{n}^{\prime}$, $f_{n}^{\prime}, g_{n}^{\prime}, h_{n}^{\prime}, \dot{\lambda}_{n}, \dot{\mu}_{n}, \dot{g_{n}}, \dot{h_{n}}, \dot{\lambda_{n}}{ }^{\prime}, \rho_{n}^{\prime}, p_{n}^{\prime}, \dot{j}_{n}^{\prime}, \tilde{\mu_{n}}{ }^{\prime}$, are uniformly bounded in the $L^{\infty}$-norm by a function of $t$ on $\left[T_{1}^{*}, 1\right]$ with $T_{1}^{*}=\max \left(T_{1}, T_{2}\right)$.

In order to prove the convergence of the iterates in the following proposition, we introduce auxiliary variables $\tilde{g}_{n}$ and $\tilde{h}_{n}$ defined by $\tilde{g}_{n}=g_{n} e^{-\mu_{n}}$, $\tilde{h}_{n}=h_{n} e^{-\lambda_{n}}$, for $n \in \mathbb{N}$. 
Proposition 4.3 Let $\left[T_{3}, 1\right] \subset\left[T_{2}, 1\right]$, be an arbitrary compact subset on which the previous estimates hold. Then on such an interval, the iterates converge uniformly.

Proof: Define for $t \in\left[T_{3}, 1\right]$ :

$$
\begin{aligned}
\alpha_{n}(t) & :=\sup \left\{\left\|\left(f_{n+1}-f_{n}\right)(s)\right\|+\left\|\left(\tilde{g}_{n+1}-\tilde{g}_{n}\right)(s)\right\|+\left\|\left(\tilde{h}_{n+1}-\tilde{h}_{n}\right)(s)\right\|\right. \\
& \left.+\left\|\left(\lambda_{n+1}-\lambda_{n}\right)(s)\right\|+\left\|\left(\mu_{n+1}-\mu_{n}\right)(s)\right\| ; \quad t \leq s \leq 1\right\}
\end{aligned}
$$

and let $C$ denote a constant which may depend on the functions $z_{1}$ and $z_{2}$ introduced previously. If we consider the new quantities

$$
\tilde{X}_{n}=\left(\tilde{g}_{n+1}-\tilde{g}_{n}\right)-\left(\tilde{h}_{n+1}-\tilde{h}_{n}\right) ; \quad \tilde{Y}_{n}=\left(\tilde{g}_{n+1}-\tilde{g}_{n}\right)+\left(\tilde{h}_{n+1}-\tilde{h}_{n}\right),
$$

then we obtain by subtracting the system (4.8)-(4.9) written for $n+1$ and $n$, the new system

$$
\begin{aligned}
& D_{n}^{+} \tilde{X}_{n}=a_{n} \tilde{X}_{n-1}+b_{n} \tilde{Y}_{n-1}+F_{n} \\
& D_{n}^{-} \tilde{Y}_{n}=b_{n} \tilde{X}_{n-1}+c_{n} \tilde{Y}_{n-1}+G_{n}
\end{aligned}
$$

where

$$
\begin{aligned}
F_{n} & =\left(a_{n}-a_{n-1}+b_{n}-b_{n-1}\right) \tilde{g}_{n-1}+\left(a_{n-1}-a_{n}+b_{n}-b_{n-1}\right) \tilde{h}_{n-1} \\
& +\left(e^{-\mu_{n-1}}-e^{-\mu_{n}}\right)\left(\dot{\tilde{g}}_{n}-\dot{\tilde{h}}_{n}\right)+\left(e^{-\lambda_{n-1}}-e^{-\lambda_{n}}\right)\left(\tilde{g}_{n}^{\prime}-\tilde{h}_{n}^{\prime}\right)
\end{aligned}
$$

and substitute in $F_{n}, \tilde{h}_{n}^{\prime}$ and $\tilde{g}_{n}^{\prime}$ respectively by $-\tilde{h}_{n}^{\prime}$ and $-\tilde{g}_{n}^{\prime}$ to obtain $G_{n}$. Now let

$$
\theta_{n}(t)=\sup \left\{\left|\tilde{g}_{n+1}-\tilde{g}_{n}\right|+\left|\tilde{h}_{n+1}-\tilde{h}_{n}\right| ; r \in \mathbb{R}\right\}
$$

Thus similarly to 4.11), we have :

$$
\theta_{n}(t) \leq 3 \int_{t}^{1}\left(m_{n-1}(s) \theta_{n-1}(s)+\sup \left\{\left|F_{n}(s, r)\right|+\left|G_{n}(s, r)\right| ; r \in \mathbb{R}\right\}\right) d s
$$

Using the mean value theorem to express the differences $e^{-\mu_{n}}-e^{-\mu_{n-1}}$, $e^{-\lambda_{n}}-e^{-\lambda_{n-1}}$ and remark 4.2, then (4.28) gives

$$
\left|\tilde{g}_{n+1}-\tilde{g}_{n}\right|+\left|\tilde{h}_{n+1}-\tilde{h}_{n}\right| \leq C \int_{t}^{1}\left(\alpha_{n-1}+\left|\tilde{\mu}_{n}-\tilde{\mu}_{n-1}\right|+\left|\dot{\lambda}_{n}-\dot{\lambda}_{n-1}\right|\right)(s) d s
$$

The expressions of $\rho_{n}, p_{n}, j_{n}$ yield, using Proposition 4.1, that

$$
\left|\rho_{n+1}-\rho_{n}\right|(t),\left|p_{n+1}-p_{n}\right|(t),\left|j_{n+1}-j_{n}\right|(t) \leq C \alpha_{n}(t)
$$

From (4.5) and (4.7), we have respectively

$$
\left|\dot{\lambda}_{n}-\dot{\lambda}_{n-1}\right|(t) \leq C \alpha_{n-1}(t)
$$




$$
\left|\tilde{\mu}_{n}-\tilde{\mu}_{n-1}\right|(t) \leq C \alpha_{n-1}(t)
$$

Using the two previous inequalities, (4.29) gives

$$
\left(\left|\tilde{g}_{n+1}-\tilde{g}_{n}\right|+\left|\tilde{h}_{n+1}-\tilde{h}_{n}\right|\right)(t) \leq C \int_{t}^{1} \alpha_{n-1}(s) d s
$$

By the mean value theorem, (4.4) gives :

$$
\left|\mu_{n+1}-\mu_{n}\right|(t) \leq C \int_{t}^{1} \alpha_{n}(s) d s
$$

(4.6) gives :

$$
\left|\lambda_{n+1}-\lambda_{n}\right|(t) \leq C \int_{t}^{1} \alpha_{n}(s) d s
$$

Following step 3 in the proof of theorem 3.1 in [13, we have

$$
\left|(R, W)_{n+1}-(R, W)_{n}\right|(1, t, r, w, F) \leq C \int_{t}^{1} \alpha_{n-1}(s) d s
$$

This implies using (4.2) and the mean value theorem

$$
\left|\left(f_{n+1}-f_{n}\right)(t)\right| \leq C\left|\partial_{r, w} \stackrel{\circ}{f}\right| \int_{t}^{1} \alpha_{n-1}(s) d s
$$

Adding (4.32), (4.33), (4.34), 4.35), then

$$
\alpha_{n}(t) \leq C \int_{t}^{1}\left(\alpha_{n}(s)+\alpha_{n-1}(s)\right) d s ; n \geq 1 .
$$

By Gronwall's inequality $\alpha_{n}(t) \leq C \int_{t}^{1} \alpha_{n-1}(s) d s$;

and by induction

$$
\alpha_{n}(t) \leq C^{n+1} \frac{(1-t)^{n}}{n !} \leq \frac{C^{n+1}}{n !} \text { for } n \in \mathbb{N}, t \in\left[T_{3}, 1\right]
$$

Since the series $\sum \frac{C^{n+1}}{n !}$ converges, we deduce the convergence of $\sum \alpha_{n}$ which implies that $\alpha_{n} \rightarrow 0$ for $n \rightarrow \infty$. Every difference term which appears in $\alpha_{n}$, converges to zero. We deduce the uniform convergence of

$$
f_{n}, \lambda_{n}, \mu_{n}, \tilde{g}_{n}, \tilde{h}_{n}, \dot{\lambda}_{n}, \dot{\mu}_{n}, \tilde{\mu}_{n}, \rho_{n}, p_{n}, j_{n}
$$

And in $L^{\infty}-$ norm, $\lambda_{n} \rightarrow \lambda ; \quad \mu_{n} \rightarrow \mu ; \tilde{\mu}_{n} \rightarrow \tilde{\mu} ; \quad f_{n} \rightarrow f ; \quad \tilde{g}_{n} \rightarrow \tilde{g} ; \tilde{h}_{n} \rightarrow \tilde{h} . \square$

It remains to show that the limits $\tilde{g}, \tilde{h}, f, \lambda, \mu$ are $C^{1}, f$ solves the Vlasov equation (1.2), $\lambda, \mu$ solve the field equations (1.3)-(1.4), and to show the existence of function $\phi$ that solves the wave equation (1.7). This is the subject of the next theorem. 
Theorem 4.4 (local existence) Let $\stackrel{\circ}{f} \in C^{1}\left(\mathbb{R}^{2} \times[0, \infty[)\right.$ with $\stackrel{\circ}{f}(r+1, w, F)=\stackrel{\circ}{f}(r, w, F)$ for $(r, w, F) \in \mathbb{R}^{2} \times[0, \infty[, \stackrel{\circ}{f} \geq 0$, and

$$
\begin{gathered}
W_{0}:=\sup \{|w| \mid(r, w, F) \in \operatorname{supp} f\}<\infty \\
F_{0}:=\sup \{F \mid(r, w, F) \in \operatorname{supp} \stackrel{\circ}{f}\}<\infty
\end{gathered}
$$

Let $\stackrel{\circ}{\lambda}, \psi \in C^{1}(\mathbb{R}), \stackrel{\circ}{\mu}, \stackrel{\circ}{\phi} \in C^{2}(\mathbb{R})$ with $\stackrel{\circ}{\lambda}(r)=\stackrel{\circ}{\lambda}(r+1), \stackrel{\circ}{\mu}(r)=\stackrel{\circ}{\mu}(r+1)$, $\stackrel{\circ}{\phi}(r)=\stackrel{\circ}{\phi}(r+1)$ and

$$
\stackrel{\circ}{\mu}^{\prime}(r)=-4 \pi e^{\stackrel{\circ}{\lambda}+\stackrel{\circ}{\mu}} j(r), \quad r \in \mathbb{R}
$$

Then there exists a unique, left maximal, regular solution $(f, \lambda, \mu, \phi)$ of system 1.2)-1.11) with $(f, \lambda, \mu, \phi)(1)=(\stackrel{\circ}{f}, \stackrel{\circ}{\lambda}, \stackrel{\circ}{\mu}, \stackrel{\circ}{\phi})$ and $\dot{\phi}(1)=\psi$ on a time interval ]$T, 1]$ with $T \in[0,1[$.

Proof : Consider the sequences of iterates constructed at the begining of this section and the limit obtained in the above proposition. We need the uniform convergence of the derivatives of these iterates.

In what follows, we fix $T_{4} \in\left[T_{2}, 1\right], t \in\left[T_{4}, 1\right],|w|<U, F<F_{0}, t \leq s \leq 1$.

Step 1: Convergence of $\left(\partial_{r} f_{n}\right)$ and $\left(\partial_{w} f_{n}\right)$.

Following step 4 in the proof of theorem 3.1 in [13], and using (4.36), we can establish with minor changes, using Proposition 4.1, that if we set:

$$
\begin{gathered}
\xi_{n}(s)=e^{\left(\lambda_{n}-\mu_{n}\right)(s, r)} \partial R_{n}(s, t, r, w, F) \\
\eta_{n}(s)=\partial W_{n}(s)+\left(\sqrt{1+w^{2}+F / s^{2}} e^{\lambda_{n}-\mu_{n}} \dot{\lambda}_{n}\right) \partial R_{n}(s)
\end{gathered}
$$

in which $\partial$ stands for $\partial_{r}$ or $\partial_{w}$ and $s \mapsto\left(R_{n}(s), W_{n}(s)\right)$ the indicated solution of the characteristic system associated to equation (4.3) in $f_{n}$, and then: $\forall \epsilon>0$, $\exists N \in \mathbb{N}$ such that we have, for $n>N$ :

$$
\left(\left|\xi_{n+1}-\xi_{n}\right|+\left|\eta_{n+1}-\eta_{n}\right|\right)(s) \leq C \epsilon+C \int_{s}^{1}\left(\left|\xi_{n+1}-\xi_{n}\right|+\left|\eta_{n+1}-\eta_{n}\right|\right)(\tau) d \tau
$$

in which $C>0$ stands, as in what follows, for a constant that may change from line to line. (4.39) implies by Gronwall's lemma, that $\left(\xi_{n}\right)$ and $\left(\eta_{n}\right)$ converge uniformly. Now, since the transformation $\left(\partial R_{n}, \partial W_{n}\right) \mapsto\left(\xi_{n}, \eta_{n}\right)$ defined by (4.37)-(4.38) is invertible with convergent coefficients, this implies the convergence of $\partial_{r, w}\left(R_{n}, W_{n}\right)$ and, given (4.2), the convergence of $\left(\partial_{r} f_{n}\right)$ and $\left(\partial_{w} f_{n}\right)$. Step 2: convergence of $\left(\lambda_{n}^{\prime}\right),\left(\mu_{n}^{\prime}\right),\left(\tilde{\mu}_{n}^{\prime}\right),\left(\tilde{g}_{n}^{\prime}\right),\left(\tilde{h}_{n}^{\prime}\right)$. We set

$$
\begin{array}{r}
\gamma_{n}(t)=\sup \left\{\left|\xi_{n+1}-\xi_{n}\right|(s)+\left|\eta_{n+1}-\eta_{n}\right|(s)+\left\|\left(\mu_{n+1}^{\prime}-\mu_{n}^{\prime}\right)(s)\right\|\right. \\
\left.+\left\|\left(\lambda_{n+1}^{\prime}-\lambda_{n}^{\prime}\right)(s)\right\|+\left\|\left(\tilde{g}_{n+1}^{\prime}-\tilde{g}_{n}^{\prime}\right)(s)\right\|+\left\|\left(\tilde{h}_{n+1}^{\prime}-\tilde{h}_{n}^{\prime}\right)(s)\right\| ; t \leq s \leq 1\right\}
\end{array}
$$


Now since $\left(\mu_{n}\right),\left(\tilde{\mu}_{n}\right),\left(\dot{\lambda}_{n}\right),\left(\tilde{g}_{n}\right),\left(\tilde{h}_{n}\right),\left(\rho_{n}\right),\left(j_{n}\right)$ converge uniformly, we take the above integer $N$ sufficiently large so that we have for $n>N$ :

$$
\begin{array}{r}
\left\|\left(\mu_{n+1}-\mu_{n}\right)(s)\right\|,\left\|\left(j_{n+1}-j_{n}\right)(s)\right\|,\left\|\left(\tilde{\mu}_{n}-\tilde{\mu}_{n-1}\right)(s)\right\|,\left\|\left(\dot{\lambda}_{n+1}-\dot{\lambda}_{n}\right)(s)\right\|, \\
\left\|\left(\tilde{g}_{n}-\tilde{g}_{n-1}\right)(s)\right\|,\left\|\left(\tilde{h}_{n}-\tilde{h}_{n-1}\right)(s)\right\|,\left\|\left(\rho_{n+1}-\rho_{n}\right)(s)\right\| \leq \epsilon
\end{array}
$$

A) Estimation of $\left(\lambda_{n}^{\prime}\right),\left(\mu_{n}^{\prime}\right),\left(\tilde{\mu}_{n}^{\prime}\right)$. We deduce from (4.37)-(4.38), taking $\partial=\partial_{r}$ that:

$$
\begin{gathered}
\partial R_{n}(s)=e^{\left(\mu_{n}-\lambda_{n}\right)(s, r)} \xi_{n}(s) \\
\partial W_{n}(s)=\eta_{n}(s)-\left(\sqrt{1+w^{2}+F / s^{2}} \dot{\lambda}_{n}\right) \xi_{n}(s)
\end{gathered}
$$

We have, using (4.2), (4.42), 4.43)

$$
\begin{aligned}
\left\|\left(\partial_{r} f_{n+1}-\partial_{r} f_{n}\right)(s)\right\| & \leq\left\|\partial_{r, w} f\right\|\left(\left|\partial_{r} R_{n+1}-\partial_{r} R_{n}\right|+\left|\partial_{r} W_{n+1}-\partial_{r} W_{n}\right|\right)(s) \\
& \leq C\left\|\partial_{r, w} f\right\|\left(\left|e^{\mu_{n+1}-\lambda_{n+1}} \xi_{n+1}-e^{\mu_{n}-\lambda_{n}} \xi_{n}\right|\right. \\
& \left.+\left|\eta_{n+1}-\eta_{n}\right|+\left|\dot{\lambda}_{n+1} \xi_{n+1}-\dot{\lambda}_{n} \xi_{n}\right|\right)(s)
\end{aligned}
$$

(4.44) gives, using (4.41) and since $\left(\lambda_{n}\right),\left(\mu_{n}\right),\left(\xi_{n}\right),\left(\dot{\lambda_{n}}\right)$ are bounded,

$$
\left\|\left(\partial_{r} f_{n+1}-\partial_{r} f_{n}\right)(s)\right\| \leq C\left(\left|\xi_{n+1}-\xi_{n}\right|+\left|\eta_{n+1}-\eta_{n}\right|\right)(s)+C \epsilon
$$

Next we have, using (4.41) and remark 4.2 :

$$
\left|\left(\tilde{g}_{n} \tilde{h}_{n}\right)^{\prime}-\left(\tilde{g}_{n-1} \tilde{h}_{n-1}\right)^{\prime}\right| \leq C \epsilon+C\left(\left|\tilde{g}_{n}^{\prime}-\tilde{g}_{n-1}^{\prime}\right|+\left|\tilde{h}_{n}^{\prime}-\tilde{h}_{n-1}^{\prime}\right|\right)
$$

Now using (4.41) and the fact that $\left(\tilde{g}_{n}\right),\left(\tilde{h}_{n}\right),\left(\tilde{g}_{n}^{\prime}\right), \tilde{h}_{n}^{\prime}$ are bounded, we obtain

$$
\left|\frac{1}{2}\left(\tilde{g}_{n}^{2}+\tilde{h}_{n}^{2}\right)^{\prime}-\frac{1}{2}\left(\tilde{g}_{n-1}^{2}+\tilde{h}_{n-1}^{2}\right)^{\prime}\right| \leq C\left(\left|\tilde{g}_{n}^{\prime}-\tilde{g}_{n-1}^{\prime}\right|+\left|\tilde{h}_{n}^{\prime}-\tilde{h}_{n-1}^{\prime}\right|\right)+C \epsilon
$$

We then deduce, from the expressions of $\rho_{n}, p_{n}, j_{n}$ and using (4.40), (4.45), (4.46), (4.47), (4.41)

$\left\|\left(\rho_{n+1}^{\prime}-\rho_{n}^{\prime}\right)(s)\right\|,\left\|\left(p_{n+1}^{\prime}-p_{n}^{\prime}\right)(s)\right\|,\left\|\left(j_{n+1}^{\prime}-j_{n}^{\prime}\right)(s)\right\| \leq C \epsilon+C\left(\gamma_{n}+\gamma_{n-1}\right)(s)$

Concerning $\left(\mu_{n}^{\prime}\right)$, we obtain by taking the derivative of (4.4) with respect to $r$, subtracting the relations written for $n+1$ and $n$, after using (4.41), (4.48) and the fact that $\mu_{n}, \mu_{n}^{\prime}$ are bounded :

$$
\left\|\left(\mu_{n+1}^{\prime}-\mu_{n}^{\prime}\right)(s)\right\| \leq C \epsilon+C \int_{s}^{1}\left(\gamma_{n-1}(\tau)+\gamma_{n}(\tau)\right) d \tau
$$

Concerning $\left(\lambda_{n}^{\prime}\right)$, if we take the derivative of (4.5) with respect to $r$, we have

$$
\dot{\lambda}_{n}^{\prime}=\left(8 \pi t \mu_{n}^{\prime} \rho_{n}+4 \pi t \rho_{n}^{\prime}\right) e^{2 \mu_{n}}-k \frac{\mu_{n}^{\prime}}{t} e^{2 \mu_{n}}
$$


We first deduce that $\overrightarrow{\lambda_{n}}$ ' is bounded. Next, subtracting (4.50) written for $n+1$ and $n$, we obtain, using (4.40), (4.41), (4.48), and since $\left(\mu_{n}\right),\left(\rho_{n}\right),\left(\mu_{n}^{\prime}\right)$ are bounded

$$
\left\|\left(\dot{\lambda}_{n+1}^{\prime}-\dot{\lambda}_{n}^{\prime}\right)(s)\right\| \leq C \epsilon+C\left(\gamma_{n-1}(s)+\gamma_{n}(s)\right)
$$

Now, from (4.6), using (4.51) and integrating over $[s, 1]$, we obtain :

$$
\left\|\left(\lambda_{n+1}^{\prime}-\lambda_{n}^{\prime}\right)(s)\right\| \leq C \epsilon+C \int_{s}^{1}\left(\gamma_{n-1}(\tau)+\gamma_{n}(\tau)\right) d \tau
$$

We will also need to bound $\tilde{\mu}_{n+1}^{\prime}-\tilde{\mu}_{n}^{\prime}$. If we take the derivative of (4.7) with respect to $r$, we obtain, after subtracting the expressions written for $n+1$ and $n$ and using (4.40), (4.41), (4.48):

$$
\left\|\left(\tilde{\mu}_{n+1}^{\prime}-\tilde{\mu}_{n}^{\prime}\right)(s)\right\| \leq C \epsilon+C\left(\gamma_{n-1}(s)+\gamma_{n}(s)\right)
$$

B) Estimation of $\left(\tilde{g}_{n}^{\prime}\right),\left(\tilde{h}_{n}^{\prime}\right)$. Recall that

$$
\begin{array}{r}
X_{n}=\tilde{g}_{n}-\tilde{h}_{n} ; Y_{n}=\tilde{g}_{n}+\tilde{h}_{n} ; a_{n}=\left(-\dot{\lambda}_{n}-\frac{1}{t}\right) e^{-\mu_{n}}-\tilde{\mu}_{n} e^{-\lambda_{n}} ; \\
b_{n}=-\frac{e^{-\mu_{n}}}{t} ; c_{n}=\left(-\dot{\lambda}_{n}-\frac{1}{t}\right) e^{-\mu_{n}}+\tilde{\mu}_{n} e^{-\lambda_{n}}
\end{array}
$$

Set

$$
\begin{array}{ll}
C_{n}=a_{n} X_{n}+b_{n} Y_{n} ; & \tilde{C}_{n}=\left(\lambda_{n}^{\prime}-\mu_{n}^{\prime}\right) e^{-\lambda_{n}} X_{n+1}^{\prime}+\mu_{n}^{\prime} C_{n}+C_{n}^{\prime} \\
D_{n}=b_{n} X_{n}+c_{n} Y_{n} ; & \tilde{D}_{n}=\left(\mu_{n}^{\prime}-\lambda_{n}^{\prime}\right) e^{-\lambda_{n}} Y_{n+1}^{\prime}+\mu_{n}^{\prime} D_{n}+D_{n}^{\prime}
\end{array}
$$

Then system (4.8)-(4.9) becomes

$$
\left\{\begin{array}{l}
D_{n}^{+} X_{n+1}=C_{n} \\
D_{n}^{-} Y_{n+1}=D_{n}
\end{array}\right.
$$

If we take the derivative of the above system with respect to $r$, a direct calculation shows that

$$
\begin{aligned}
& D_{n}^{+} X_{n+1}^{\prime}=\tilde{C}_{n} \\
& D_{n}^{-} Y_{n+1}^{\prime}=\tilde{D}_{n}
\end{aligned}
$$

Consider the following characteristic curves $\gamma_{n}^{1}, \gamma_{n}^{2}$ of the wave operator, starting from the point $(s, r)$, i.e for every $n$,

$$
\dot{\gamma}_{n}^{1}=e^{\lambda_{n}-\mu_{n}}, \dot{\gamma}_{n}^{2}=-e^{\lambda_{n}-\mu_{n}}, \gamma_{n}^{1}(s)=\gamma_{n}^{2}(s)=r
$$

We have $D_{n}^{+}=2 e^{-\mu_{n}} \frac{d}{d t}$ on $\gamma_{n}^{1}$ and $D_{n}^{-}=2 e^{-\mu_{n}} \frac{d}{d t}$ on $\gamma_{n}^{2}$. We then have, integrating (4.56) over $\gamma_{n}^{1}$ and (4.57) over $\gamma_{n}^{2}$, and after subtraction,

$$
\left(X_{n+1}^{\prime}-X_{n}^{\prime}\right)(s)=\frac{1}{2} \int_{s}^{1}\left[e^{\mu_{n-1}} \tilde{C}_{n-1}\left(\tau, \gamma_{n-1}^{1}(\tau)\right)-e^{\mu_{n}} \tilde{C}_{n}\left(\tau, \gamma_{n}^{1}(\tau)\right)\right] d \tau
$$




$$
\left(Y_{n+1}^{\prime}-Y_{n}^{\prime}\right)(s)=\frac{1}{2} \int_{s}^{1}\left[e^{\mu_{n-1}} \tilde{D}_{n-1}\left(\tau, \gamma_{n-1}^{2}(\tau)\right)-e^{\mu_{n}} \tilde{D}_{n}\left(\tau, \gamma_{n}^{2}(\tau)\right)\right] d \tau
$$

Since $e^{\mu_{n}}$ and $\tilde{C}_{n}$ are bounded, we have

$$
\begin{array}{r}
\left|e^{\mu_{n}} \tilde{C}_{n}\left(\tau, \gamma_{n}^{1}(\tau)\right)-e^{\mu_{n-1}} \tilde{C}_{n-1}\left(\tau, \gamma_{n-1}^{1}(\tau)\right)\right| \leq C\left[\left\|\left(e^{\mu_{n}}-e^{\mu_{n-1}}\right)(\tau)\right\|\right. \\
+\left\|\left(\tilde{C}_{n}-\tilde{C}_{n-1}\right)(\tau)\right\| \\
+\left|\tilde{C}_{n-1}\left(\tau, \gamma_{n}^{1}(\tau)\right)-\tilde{C}_{n-1}\left(\tau, \gamma_{n-1}^{1}(\tau)\right)\right| \\
\left.+\left|e^{\mu_{n-1}}\left(\tau, \gamma_{n}^{1}(\tau)\right)-e^{\mu_{n-1}}\left(\tau, \gamma_{n-1}^{1}(\tau)\right)\right|\right]
\end{array}
$$

Now integrating the relation $\dot{\gamma}_{n}^{1}-\dot{\gamma}_{n-1}^{1}=e^{\lambda_{n}-\mu_{n}}-e^{\lambda_{n-1}-\mu_{n-1}}$ over $[s, \tau]$ yields

$$
\left|\gamma_{n}^{1}-\gamma_{n-1}^{1}\right|(\tau) \leq C \sup \left\{\left\|\left(\lambda_{n}-\lambda_{n-1}\right)(t)\right\|+\left\|\left(\mu_{n}-\mu_{n-1}\right)(t)\right\|, T_{4} \leq t \leq 1\right\}
$$

we then deduce from (4.36), (4.62), the uniform continuity of $\left(e^{\mu_{n-1}}\right),\left(\tilde{C}_{n-1}\right)$ over the compact set $K=\left[T_{4}, 1\right] \times\left(\gamma_{n}^{1}\left(\left[T_{4}, 1\right]\right) \cup \gamma_{n-1}^{1}\left(\left[T_{4}, 1\right]\right)\right)$, (4.61) and (4.59)(4.60), that

$$
\begin{aligned}
& \left|X_{n+1}^{\prime}-X_{n}^{\prime}\right|(s) \leq C \epsilon+\int_{s}^{1}\left\|\left(\tilde{C}_{n}-\tilde{C}_{n-1}\right)(\tau)\right\| d \tau \\
& \left|Y_{n+1}^{\prime}-Y_{n}^{\prime}\right|(s) \leq C \epsilon+\int_{s}^{1}\left\|\left(\tilde{D}_{n}-\tilde{D}_{n-1}\right)(\tau)\right\| d \tau
\end{aligned}
$$

Therefore, for $n$ sufficiently large,

$$
\begin{gathered}
\left\|\left(\tilde{g}_{n+1}^{\prime}-\tilde{g}_{n}^{\prime}\right)(s)\right\|,\left\|\left(\tilde{h}_{n+1}^{\prime}-\tilde{h}_{n}^{\prime}\right)(s)\right\| \leq C \epsilon+C \int_{s}^{1}\left[\left\|\left(\tilde{C}_{n}-\tilde{C}_{n-1}\right)(\tau)\right\|\right. \\
\left.+\left\|\left(\tilde{D}_{n}-\tilde{D}_{n-1}\right)(\tau)\right\|\right] d \tau
\end{gathered}
$$

Now from (4.55), (4.41) and the fact that the sequences $\left(\dot{\lambda}_{n}\right),\left(\lambda_{n}\right),\left(\mu_{n}\right),\left(\tilde{\mu}_{n}^{\prime}\right)$ are bounded together with their first derivatives, we have

$$
\left\|\left(\tilde{C}_{n}-\tilde{C}_{n-1}\right)(\tau)\right\| \leq C \epsilon+C\left(\gamma_{n-2}(\tau)+\gamma_{n-1}(\tau)+\gamma_{n}(\tau)\right)
$$

and

$$
\left\|\left(\tilde{D}_{n}-\tilde{D}_{n-1}\right)(\tau)\right\| \leq C \epsilon+C\left(\gamma_{n-2}(\tau)+\gamma_{n-1}(\tau)+\gamma_{n}(\tau)\right)
$$

Therefore, we deduce from (4.65) that

$\left\|\left(\tilde{g}_{n+1}^{\prime}-\tilde{g}_{n}^{\prime}\right)(s)\right\|,\left\|\left(\tilde{h}_{n+1}^{\prime}-\tilde{h}_{n}^{\prime}\right)(s)\right\| \leq C \epsilon+C \int_{s}^{1}\left(\gamma_{n-2}(\tau)+\gamma_{n-1}(\tau)+\gamma_{n}(\tau)\right) d \tau$

C) Convergence of $\left(\lambda_{n}^{\prime}\right),\left(\mu_{n}^{\prime}\right),\left(\tilde{\mu}_{n}^{\prime}\right),\left(\tilde{g}_{n}^{\prime}\right),\left(\tilde{h}_{n}^{\prime}\right)$. Add inequalities (4.39), (4.49), (4.52) and (4.66) and take the supremum over $s \in[t, 1]$ to obtain using (4.40)

$$
\gamma_{n}(t) \leq C \epsilon+C \int_{t}^{1}\left(\gamma_{n-2}(s)+\gamma_{n-1}(s)+\gamma_{n}(s)\right) d s
$$


Define $\Gamma_{n}(t)=\sup \left\{\gamma_{m}, m \leq n\right\}$; then $\left(\Gamma_{n}\right)$ is an increasing sequence and (4.67) gives

$$
\Gamma_{n}(t) \leq C \epsilon+C \int_{t}^{1} \Gamma_{n}(s) d s
$$

And by Gronwall's lemma,

$$
\Gamma_{n}(t) \leq C \epsilon, \quad n>N, \quad t \in\left[T_{4}, 1\right]
$$

We then deduce that $\left(\Gamma_{n}\right)$ converges uniformly to 0 , and from 4.40)-4.53), $\left(\lambda_{n}^{\prime}\right)$, $\left(\mu_{n}^{\prime}\right),\left(\tilde{g}_{n}^{\prime}\right),\left(\tilde{h}_{n}^{\prime}\right),\left(\tilde{\mu}_{n}^{\prime}\right)$ converge uniformly on $\left[T_{4}, 1\right]$. We deduce from system (4.8)-(4.9), the uniform convergence of $\left(\dot{\tilde{g}}_{n}\right)$ and $\left(\dot{\tilde{h}}_{n}\right)$. The regularity of $f, \lambda, \mu$, $\tilde{g}, \tilde{h}$ (and $\tilde{\mu}$ ) follows from step 1 and step 2. Therefore $g=e^{\mu} \tilde{g}$ and $h=e^{\lambda} \tilde{h}$ are regular. Note that, using the convergence of the derivatives, we can prove that the limit $(f, \lambda, \mu, \tilde{\mu}, g, h)$ is a regular solution of (2.3), (1.3), (1.4), (2.4), (2.5), (2.6) and by Proposition 3.2 we conclude the existence of a regular function $\phi$ such that $(f, \lambda, \mu, \phi)$ is a solution of the full system (1.2)-(1.11). To end this theorem, we prove the uniqueness of the solution. Let $u_{i}=\left(f_{i}, \lambda_{i}, \mu_{i}, \phi_{i}\right)$, $i=1,2$ be two regular solutions of the Cauchy problem for the same initial data $(\stackrel{\circ}{f}, \stackrel{\circ}{\lambda}, \stackrel{\circ}{\mu}, \stackrel{\circ}{\phi}, \psi)$ at $t=1$. Using the fact that $u_{i}$ is a solution of the system, one proceeds similarly as to prove the convergence of iterates to obtain

$$
\alpha(t) \leq C \int_{t}^{1} \alpha(s) d s
$$

where

$$
\begin{aligned}
\alpha(t) & =\sup \left\{\left\|f_{1}(s)-f_{2}(s)\right\|+\left\|\lambda_{1}(s)-\lambda_{2}(s)\right\|+\left\|\mu_{1}(s)-\mu_{2}(s)\right\|\right. \\
& \left.+\left\|\tilde{g}_{1}(s)-\tilde{g}_{2}(s)\right\|+\left\|\tilde{h}_{1}(s)-\tilde{h}_{2}(s)\right\| ; s \in[t, 1]\right\},
\end{aligned}
$$

with $\tilde{g}_{1}=\dot{\phi}_{1} e^{\mu_{1}} ; \quad \tilde{g}_{2}=\dot{\phi}_{2} e^{\mu_{2}} ; \quad \tilde{h}_{1}=\phi_{1}^{\prime} e^{\lambda_{1}} ; \quad \tilde{h}_{2}=\phi_{2}^{\prime} e^{\lambda_{2}}$. We deduce that $\alpha(t)=0$, for $t \in] 0,1]$. This implies that $f_{1}=f_{2}, \lambda_{1}=\lambda_{2}, \mu_{1}=\mu_{2}, \tilde{g}_{1}=\tilde{g}_{2}$ and $\tilde{h}_{1}=\tilde{h}_{2}$. By Proposition 3.2 we conclude that $\phi_{1}=\phi_{2}$. $\square$

Let us now prove continuation criteria for $t$ decreasing.

Theorem 4.5 Let $(\stackrel{\circ}{f}, \stackrel{\circ}{\lambda}, \stackrel{\circ}{\mu}, \stackrel{\circ}{\phi}, \psi)$ be initial data as in theorem 4.4. If $k=-1$, assume that $\stackrel{\circ}{\mu}<0$. Let $(f, \lambda, \mu, \phi)$ be a solution of [1.2)-1.11) on a left maximal interval of existence $] T, 1]$ for which

$$
\sup \{|w| \mid(t, r, w, F) \in \operatorname{supp} f\}<\infty
$$

1. If $\left.\left.\sup \left\{\left(e^{-2 \mu} \dot{\phi}^{2}+e^{-2 \lambda} \phi^{2}\right)(t, r) ; t \in\right] T, 1\right]\right\}<\infty$ then $T=0$.

2. If $\sup \left\{\left(e^{-2 \mu} \dot{\phi}^{2}+e^{-2 \lambda} \phi^{2}\right)(1, r)\right\}$ is sufficiently small then $T=0$.

Proof : Let $(f, \lambda, \mu, \tilde{\mu}, g, h)$ be a left maximal solution of the auxiliary system (2.3), (1.3), (1.4), (2.4), (2.5), (2.6) with existence interval ]T, 1]. By Proposition 3.2 there exists $\phi$ such that $(f, \lambda, \mu, \phi)$ solves (1.2)-(1.11). By assumption

$$
\left.\left.Q^{*}:=\sup \{|w| \mid(r, w, F) \in \operatorname{supp} f(t), t \in] T, 1\right]\right\}<\infty .
$$


We want to show that $T=0$, so let us assume that $T>0$ and take $\left.t_{1} \in\right] T, 1[$. We will show that the system has a solution with initial data $\left(f\left(t_{1}\right), \lambda\left(t_{1}\right), \mu\left(t_{1}\right), \phi\left(t_{1}\right)\right.$, $\left.\dot{\phi}\left(t_{1}\right)\right)$ prescribed at $t=t_{1}$ which exists on an interval $\left[t_{1}-\delta, t_{1}\right]$ with $\delta>0$ independent of $t_{1}$. By moving $t_{1}$ close enough to $T$ this would extend our initial solution beyond $T$, a contradiction to the initial solution being left maximal. We have proved in Proposition 4.1 that such a solution exists at least on the left maximal existence interval of the solutions $\left(z_{1}, z_{2}\right)$ of

$$
\begin{gathered}
z_{1}(t)=W\left(t_{1}\right)+K\left(t_{1}\right)+C_{1} \int_{t}^{t_{1}} \frac{1}{s}\left(1+z_{1}(s)\right)^{5} d s \\
z_{2}(t)=C_{4} \exp \left[\int_{t}^{t_{1}} C_{2}(s)\left(C_{3}+z_{2}(s)\right) d s\right],
\end{gathered}
$$

where

$$
\begin{gathered}
W\left(t_{1}\right):=\sup \left\{|w| \mid(r, w, F) \in \operatorname{supp} f\left(t_{1}\right)\right\}, \\
K\left(t_{1}\right):=\sup \left\{\left(\left|\dot{\phi}^{2}\right| e^{-2 \mu}+\left|\phi^{\prime 2}\right| e^{-2 \lambda}\right)^{\frac{1}{2}}\left(t_{1}, r\right) ; r \in \mathbb{R}\right\}, \\
A\left(t_{1}\right):=\sup \left\{\left[\left(\dot{\phi}^{\prime}-\mu^{\prime} \dot{\phi}\right)^{2} e^{-2 \mu}+\left(\phi^{\prime \prime}-\lambda^{\prime} \phi^{\prime}\right)^{2} e^{-2 \lambda}\right]\left(t_{1}, r\right) ; r \in \mathbb{R}\right\}, \\
C_{1}=\frac{C}{C_{0}}\left(1+F_{0}\right)^{2}\left(1+\left\|f\left(t_{1}\right)\right\|\right) ; C_{0}=\inf \left\{e^{-\mu\left(t_{1}, r\right)} ; r \in \mathbb{R}\right\} \\
C_{3}:=\left\|e^{-2 \mu\left(t_{1}\right)} \mu^{\prime}\left(t_{1}\right)\right\|+\left\|\lambda^{\prime}\left(t_{1}\right)\right\|+1 ; C_{4}:=A\left(t_{1}\right)+\left\|\partial_{(r, w)} f\left(t_{1}\right)\right\|
\end{gathered}
$$

and $C_{2}$ is an increasing function of $z_{1}$. Now $W\left(t_{1}\right) \leq Q^{*},\left\|f\left(t_{1}\right)\right\|=\|\stackrel{\circ}{f}\|, F_{0}$ is unchanged since $F$ is constant along characteristics, and since $t_{1}<1$, taking $t=t_{1}$ in (4.4) shows that: $C_{0}\left(\mu\left(t_{1}\right)\right) \geq C_{0}(\stackrel{\circ}{\mu})$. Thus there exists a constant $M_{1}>0$ such that

$$
\left.\left.\frac{1}{T} C_{1}\left(f\left(t_{1}\right), F_{0}, \mu\left(t_{1}\right)\right) \leq M_{1} \text { for } t_{1} \in\right] T, 1\right] .
$$

The expressions of $\rho, p, j, \dot{\lambda}, \tilde{\mu}$ show since $|w| \leq Q^{*}$, that

$$
|\rho(s, r)|,|p(s, r)|,|j(s, r)|,|\dot{\lambda}(s, r)|,\left|\tilde{\mu} e^{\mu-\lambda}(s, r)\right| \leq C+(K(s))^{2}
$$

Consider now statement 1 of the theorem. In that case $K$ is bounded on ]$T, 1]$. We proceed as in step 6 of the proof of theorem 3.1 in 13 to prove that $\partial_{(r, w)} f$ is uniformly bounded on $\left.] T, 1\right]$. Let $\mu^{\prime}=\tilde{\mu}$; consider the relations

$$
\mu^{\prime}=-4 \pi t e^{\lambda+\mu} j ; \quad \mu^{\prime \prime}=-\left[\left(\lambda^{\prime}+\mu^{\prime}\right) j+j^{\prime}\right] 4 \pi t e^{\lambda+\mu}
$$




$$
\dot{\lambda}^{\prime}=e^{2 \mu}\left(8 \pi t \mu^{\prime} \rho+\frac{k \mu^{\prime}}{t}+4 \pi t \rho^{\prime}\right) ; \lambda^{\prime}=\stackrel{\circ}{\lambda}^{\prime}+\int_{1}^{t} \dot{\lambda}^{\prime}(s, r) d s
$$

We bound $\rho^{\prime}, j^{\prime}$ by quantities which depend on $\partial_{r} f$ and $A(s)$. Since $\partial_{r} f, \rho$ and $j$ are bounded, we deduce from the above relations that $\mu^{\prime \prime}, \dot{\lambda}^{\prime}$ and consequently $v(s)$ and $h(s)$ (see Proposition 2.5) are bounded each by $A(s)$. Using inequality (2.12) and the fact that $K(s)$ is bounded, we obtain

$$
A(t) \leq A_{0}+C \int_{t}^{1}(A(s)+1) d s
$$

And we deduce that $\left.\left.A(t) \leq A^{*}=\left(1+A_{0}\right) e^{C} ; t \in\right] T, 1\right]$. Therefore

$$
\left.\left.D:=\sup \left\{\left\|\partial_{(r, w)} f(t)\right\|+A(t) \mid t \in\right] T, 1\right]\right\}<\infty .
$$

From

$$
\mu^{\prime}(t, r)=\frac{e^{2 \mu}}{t}\left(\stackrel{\circ}{\mu}^{\prime}(r) e^{-2 \stackrel{\circ}{\mu}}+4 \pi \int_{1}^{t} p^{\prime}(s, r) s^{2} d s\right),
$$

(4.68), 4.69) and since $K(t)$ and $A(t)$ are bounded, $p^{\prime}$ is bounded and we obtain a uniform bound $C_{3}\left(\mu\left(t_{1}\right), \lambda\left(t_{1}\right)\right) \leq M_{3}$. Let $y_{2}$ be the left maximal solution of

$$
y_{2}(t)=D \exp \left[\int_{t}^{t_{1}} C_{2}^{*}(s)\left(M_{3}+y_{2}(s)\right) d s\right],
$$

where $C_{2}^{*}$ depends on $y_{1}$ in the same way as $C_{1}$ depends on $z_{1} . y_{2}$ exists on an interval $\left[t_{1}-\alpha, t_{1}\right]$ with $\alpha>0$ independent of $t_{1}$. If we choose $t_{1}$ such that $T>t_{1}-\alpha$ then $z_{1}$ is bounded $z_{2} \leq y_{2}$ by construction. In particular, $z_{2}$ exists on $I \subset\left[t_{1}-\alpha, t_{1}\right]$, and the proof of part 1 of the theorem is complete.

Consider now statement 2. We deduce by Gronwall's lemma applied to (2.9) and the bounds given above that

$$
K(t) \leq K_{0} \exp \int_{t}^{1} C\left(1+(K(s))^{2}\right) d s
$$

Therefore, for initial data sufficiently small, $K(t) \leq K^{*}=\frac{2 K_{0} e^{C}}{\sqrt{1+K_{0}^{2}}}$ for $\left.\left.t \in\right] T, 1\right]$; as one can see by solving explicitly the associated integral equation. Thus statement 2 follows from statement 1 .

Remark 4.6 Initial data can be chosen so that the smallness condition above is satisfied. The functions $\phi, \psi$ and $\stackrel{\circ}{\lambda}$ can be chosen freely. Thus it can be arranged that the data are small provided that $\stackrel{\circ}{\mu}$ is not too negative. There is no problem controlling $\stackrel{\circ}{\mu}$ since in the construction of data we have the freedom to add any constant to $e^{-\stackrel{\circ}{\mu}}$.

We prove in the next theorem, the analogue of theorems 4.4 and 4.5 for $t \geq 1$. 
Theorem 4.7 Let $(\stackrel{\circ}{f}, \stackrel{\circ}{\lambda}, \stackrel{\circ}{\mu}, \stackrel{\circ}{\phi}, \psi)$ be initial data as in Theorem 4.4 . Then there exists a unique, right maximal, regular solution $(f, \lambda, \mu, \phi)$ of (1.2)-(1.11) with $(f, \lambda, \mu, \phi, \dot{\phi})(1)=(\stackrel{\circ}{f}, \stackrel{\circ}{\lambda}, \stackrel{\circ}{\mu}, \stackrel{\circ}{\phi}, \psi)$ on a time interval $[1, T[$ with $T \in] 1, \infty]$. If

$$
\begin{aligned}
& \sup \{|w| \mid(t, r, w, F) \in \operatorname{supp} f\}<\infty ; \\
& \sup \left\{e^{2 \mu(t, r)} \mid r \in \mathbb{R}, t \in[1, T[\}<\infty\right.
\end{aligned}
$$

and

$$
K(t)<\infty
$$

then $T=\infty$.

Proof : We give only those parts of the proof which differ from the proof of Theorem 4.4 for $t \leq 1$. The iterates are defined in the same way as before, except that now (4.4) is used to define $\mu_{n}$ only on the interval $\left[1, T_{n}[\right.$, where

$T_{n}:=\sup \{\tau \in] 1, T_{n-1}\left[\mid \frac{e^{-2 \stackrel{\circ}{\mu}(r)}}{t}-\frac{8 \pi}{t} \int_{1}^{t} s^{2} p_{n}(s, r) d s>0, r \in \mathbb{R}, t \in[1, \tau]\right\}$,

$\left[1, T_{n-1}\left[\right.\right.$ being the existence interval of the previous iterates and $T_{0}=\infty$. Define:

$$
\begin{gathered}
Q_{n}(t):=\sup \left\{|w|, \quad(r, w, F) \in \operatorname{supp} f_{n}(t)\right\}, t \in\left[1, T_{n}[\right. \\
E_{n}(t):=\sup \left\{s e^{2 \mu_{n}(s, r)} \mid r \in \mathbb{R}, 1 \leq s \leq t\right\}
\end{gathered}
$$

we obtain the estimates

$$
\begin{gathered}
\sqrt{1+w^{2}+F / t^{2}} \leq \sqrt{1+\left(Q_{n}(t)\right)^{2}+F_{0}} \leq\left(1+F_{0}\right)\left(1+Q_{n}(t)\right) ; \\
\left\|\rho_{n}(t)\right\|,\left\|p_{n}(t)\right\|,\left\|j_{n}(t)\right\| \leq \frac{C^{*}}{t}\left(1+Q_{n}(t)\right)^{2}+\left(K_{n-1}(t)\right)^{2} ;
\end{gathered}
$$

and

$$
\left|e^{\mu_{n}-\lambda_{n}} \tilde{\mu}_{n}(t, r)\right|+\left|\dot{\lambda}_{n}(t, r)\right| \leq C^{*}\left(1+Q_{n}(t)\right)^{2}+\left(1+K_{n-1}(t)\right)^{2}\left(1+E_{n}(t)\right) .
$$

where $C^{*}=C\left(1+F_{0}\right)^{2}(1+\|\stackrel{\circ}{f}\|)$. Thus, we have similarly to (4.16):

$Q_{n+1}(t) \leq W_{0}+C^{*} \int_{1}^{t}\left(1+Q_{n}(s)\right)^{2}\left(1+E_{n}(s)\right)\left(1+K_{n-1}(s)\right)^{2}\left(1+Q_{n+1}(s)\right) d s$.

and similarly to (4.18):

$$
K_{n+1}(t) \leq K_{0}+C^{*} \int_{1}^{t}\left(1+Q_{n}(s)\right)^{2}\left(1+K_{n-1}(s)\right)^{2} K_{n}(s)\left(1+E_{n}(s)\right) d s .
$$


We deduce from the field equation (1.4) that

$$
\left(2 \dot{\mu}_{n} e^{2 \mu_{n}}\right) t=e^{2 \mu_{n}}+k e^{4 \mu_{n}}+8 \pi\left(t e^{2 \mu_{n}}\right)^{2} p_{n}
$$

Integrating over $[1, s]$ and using integration by parts for the left hand side yields the following estimate, since (4.70) holds,

$$
E_{n}(t) \leq\left\|e^{2 \stackrel{\circ}{\mu}}\right\|+C^{*} \int_{1}^{t}\left(1+Q_{n}(s)\right)^{2}\left(1+E_{n}(s)\right)^{2}\left(1+K_{n-1}(s)\right)^{2} d s .
$$

Reasoning in the same way as in the proof of Proposition 4.1. we can say the differential inequalities (4.71), (4.72), (4.73) allow us to estimate $Q_{n}, K_{n}$ and $E_{n}$ against the solution $z_{1}, z_{2}$ and $z_{3}$ of the system

$$
\begin{gathered}
z_{1}(t)=W_{0}+C^{*} \int_{1}^{t}\left(1+z_{1}(s)\right)^{3}\left(1+z_{2}(s)\right)^{2}\left(1+z_{3}(s)\right) d s, \\
\left.z_{2}(t)=K_{0}+C^{*} \int_{1}^{t}\left(1+z_{1}(s)\right)^{2}\left(1+z_{2}(s)\right)^{3}\right]\left(1+z_{3}(s)\right) d s, \\
z_{3}(t)=\left\|e^{2 \stackrel{\circ}{\mu}}\right\|+C^{*} \int_{1}^{t}\left(1+z_{1}(s)\right)^{2}\left(1+z_{2}(s)\right)^{2}\left(1+z_{3}(s)\right)^{2} d s,
\end{gathered}
$$

and in particular $T_{n} \geq T$ where $[1, T$ [is the right maximal existence interval of $\left(z_{1}, z_{2}, z_{3}\right)$. One can now establish a bound on first order derivatives of the iterates in the same way as in the proof of Proposition 4.1 and obtains a local solution on a right maximal existence interval which is extendible if the quantities $Q(t), E(t)=\left\|t e^{2 \mu(t)}\right\|$ and $K(t)$ can be bounded.

Theorem 4.8 Let $(f, \lambda, \mu, \phi)$ be a right maximal regular solution obtained in Theorem 4.7. Assume that

$$
\sup \{|w| \mid(t, r, w, F) \in \operatorname{supp} f\}<\infty ;
$$

and

$$
\sup \left\{e^{2 \mu(t, r)} \mid r \in \mathbb{R}, t \in[1, T[\}<C<\infty ;\right.
$$

then $\quad T=\infty$.

Proof : We deduce from system (2.1)-(2.2):

$$
\begin{aligned}
& D^{+} X^{2}=2 a X^{2}+2 b X Y \\
& D^{-} Y^{2}=2 b X Y+2 c Y^{2}
\end{aligned}
$$

On the characteristic curves of the wave equation, we obtain

$$
\frac{d}{d t} X^{2}\left(t, \gamma_{1}(t)\right)=e^{\mu}\left(a X^{2}+b X Y\right)\left(t, \gamma_{1}(t)\right)
$$




$$
\frac{d}{d t} Y^{2}\left(t, \gamma_{2}(t)\right)=e^{\mu}\left(b X Y+c Y^{2}\right)\left(t, \gamma_{2}(t)\right)
$$

From (4.74), we have:

$$
\begin{aligned}
\frac{d}{d t} X^{2}\left(t, \gamma_{1}(t)\right) & =\left(-\dot{\lambda}-\mu^{\prime} e^{\mu-\lambda}-\frac{1}{t}\right) X^{2}-\frac{X Y}{t} \\
& =4 \pi t e^{2 \mu}(j-\rho) X^{2}+\frac{1+k e^{2 \mu}}{2 t} X^{2}-\frac{1}{t} X^{2}-\frac{X Y}{t} \\
& \leq\left(\frac{-1}{2 t}+\frac{k e^{2 \mu}}{2 t}\right) X^{2}+\frac{X^{2}+Y^{2}}{2 t} \quad \text { since } \quad j-\rho<0 ; \\
& \leq\left(\frac{C k}{2 t} X^{2}+\frac{1}{2 t} Y^{2}\right)\left(t, \gamma_{1}(t)\right)
\end{aligned}
$$

Since $j+\rho>0$, we deduce as above, from 4.75), the estimate

$$
\frac{d}{d t} Y^{2}\left(t, \gamma_{2}(t)\right) \leq\left(\frac{1}{2 t} X^{2}+\frac{C k}{2 t} Y^{2}\right)\left(t, \gamma_{2}(t)\right)
$$

After integrating these two inequalities over $[1, t]$ and taking the maximum over space, we obtain :

$$
\begin{aligned}
K(t)^{2} & \leq K(1)^{2}+\int_{1}^{t}\left(\frac{1}{2 s}+\frac{C k}{2 s}\right) K(s)^{2} d s \\
& \leq K(1)^{2}+\int_{1}^{t}\left(\frac{1+C k}{2 s}\right) K(s)^{2} d s
\end{aligned}
$$

We deduce by Gronwall's lemma that:

$$
K(t) \leq K(1) t^{\frac{1+C k}{4}}, \quad \text { for } t \in[1, T[
$$

And we conclude by Theorem 4.7 that $T=\infty$.

\section{$5 \quad$ Future global existence in a particular case}

In the case $k=1$, there is no global existence in the future since this already fails without a scalar field, as shown in [13] and 14. Now we prove in the special case where there is only a scalar field (i.e. $f$ is identically zero), the existence of the solution for all $t \geq 1$ for $k=0$ or $k=-1$.

Theorem 5.1 Suppose that $f=0$ and let $(\lambda, \mu, \phi)$ be a right maximal regular solution obtained in Theorem 4.7 T $T=\infty$ for $k=0$ or $k=-1$.

Proof : we establish a series of estimates which will result in an upper bound on $\mu$ and will therefore prove that $T=\infty$. Similar estimates were used in 3. Unless otherwise specified in what follows constants denoted by $C$ will be positive, may depend on the initial data and may change their value from line 
to line. Firstly, integration of (3.7) (where $\bar{p}$ is replaced by $p$ ) with respect to $t$ and the fact that $p$ is non-negative imply that

$$
e^{2 \mu(t, r)}=\left[\frac{\left(e^{-2 \mu \rho(r)}+k\right)}{t}-k-\frac{8 \pi}{t} \int_{1}^{t} s^{2} p(s, r) d s\right]^{-1} \geq \frac{t}{C-k t}, t \in[1, T[
$$

Next let us prove that

$$
\int_{0}^{1} e^{\mu+\lambda} \rho(t, r) d r \leq C t, t \in[1, T[
$$

A calculation shows that, since $\mu$ and $j$ are periodic with respect to $r$ and $\rho=p$

$$
\frac{d}{d t} \int_{0}^{1} e^{\mu+\lambda} \rho(t, r) d r=-\frac{1}{t} \int_{0}^{1} e^{\mu+\lambda}\left[2 \rho+q-\rho\left(1+k e^{2 \mu}\right)\right] d r
$$

Now $q \geq-2 \rho$ and $q+\rho \geq-\rho$ so that for $k=0$,

$$
\frac{d}{d t} \int_{0}^{1} e^{\mu+\lambda} \rho(t, r) d r \leq \frac{1}{t} \int_{0}^{1} e^{\mu+\lambda} \rho(t, r) d r
$$

and integrating this inequality with respect to $t$ yields (5.2) for $k=0$. For $k=-1$, we have, using (5.1):

$$
\begin{aligned}
\frac{d}{d t} \int_{0}^{1} e^{\mu+\lambda} \rho(t, r) d r & =-\frac{1}{t} \int_{0}^{1} e^{\mu+\lambda}\left[2 \rho+q-\rho\left(1-e^{2 \mu}\right)\right] d r \\
& \leq-\frac{1}{t} \int_{0}^{1} e^{\mu+\lambda}(\rho+q) d r-\frac{1}{C+t} \int_{0}^{1} e^{\mu+\lambda} \rho d r \\
& \leq\left(\frac{1}{t}-\frac{1}{C+t}\right) \int_{0}^{1} e^{\mu+\lambda} \rho d r \\
& \leq \frac{1}{t} \int_{0}^{1} e^{\mu+\lambda} \rho d r
\end{aligned}
$$

Integrating this inequality with respect to $t$ yields (5.2) for $k=-1$. Using (1.5), the fact that $|j| \leq \rho$ and $(5.2)$ we find

$$
\begin{aligned}
\left|\mu(t, r)-\int_{0}^{1} \mu(t, \sigma) d \sigma\right| & =\left|\int_{0}^{1} \int_{\sigma}^{r} \mu^{\prime}(t, \tau) d \tau d \sigma\right| \leq \int_{0}^{1} \int_{0}^{1}\left|\mu^{\prime}(t, \tau)\right| d \tau d \sigma \\
& \leq 4 \pi t \int_{0}^{1} e^{\mu+\lambda}|j(t, \tau)| d \tau \leq 4 \pi t \int_{0}^{1} e^{\mu+\lambda} \rho(t, \tau) d \tau
\end{aligned}
$$

that is

$$
\left|\mu(t, r)-\int_{0}^{1} \mu(t, \sigma) d \sigma\right| \leq C t^{2}, t \in[1, T[, r \in[0,1]
$$

Next we show that

$$
e^{\mu(t, r)-\lambda(t, r)} \leq C t, t \in[1, T[, r \in[0,1]
$$


Using relations $\dot{\mu}-\dot{\lambda}=4 \pi t e^{2 \mu}(p-\rho)+\frac{1+k e^{2 \mu}}{t}, p-\rho=0$ and (5.1), we obtain

$$
\begin{aligned}
\frac{\partial}{\partial t} e^{\mu-\lambda} & =(\dot{\mu}-\dot{\lambda}) e^{\mu-\lambda}=e^{\mu-\lambda} \frac{1+k e^{2 \mu}}{t} \\
& \leq\left(\frac{1}{t}+\frac{k}{C-k t}\right) e^{\mu-\lambda}
\end{aligned}
$$

and integrating this inequality with respect to $t$ yields (5.4).

We now estimate the average of $\mu$ over the interval $[0,1]$ which in combination with (5.3) will yield the desired upper bound on $\mu$. We use (5.2), 5.4) the fact that $p=\rho$ and $k e^{2 \mu} \leq 0$ :

$$
\begin{aligned}
\int_{0}^{1} \mu(t, r) d r & =\int_{0}^{1} \stackrel{\circ}{\mu}(r) d r+\int_{1}^{t} \int_{0}^{1} \dot{\mu}(s, r) d r d s \\
& \leq C+\int_{1}^{t} \frac{1}{2 s} \int_{0}^{1}\left[e^{2 \mu}\left(8 \pi s^{2} p+k\right)+1\right] d r d s \\
& =C+\frac{1}{2} \ln t+4 \pi \int_{1}^{t} \int_{0}^{1} s e^{2 \mu} \rho d r d s+\int_{1}^{t} \int_{0}^{1} \frac{k e^{2 \mu}}{2 s} d r d s \\
& \leq C+\frac{1}{2} \ln t+4 \pi \int_{1}^{t} \int_{0}^{1} s e^{\mu-\lambda} e^{\mu+\lambda} \rho d r d s \\
& \leq C+\frac{1}{2} \ln t+C \int_{1}^{t} \int_{0}^{1} s^{3} d r d s \\
& =C+\frac{1}{2} \ln t+C t^{4}
\end{aligned}
$$

with (5.3) this implies

$$
\mu(t, r) \leq C\left(1+\ln t+t^{4}+t^{2}\right) \leq C t^{4}, t \in[1, T[, r \in[0,1] .
$$

And we conclude by theorem 4.8 that $T=\infty$

Remark 5.2 we have proven that for initial data as in theorem 4.4 the corresponding solution exists for all $t \in[1, \infty[$ and satisfies the estimates (5.2)-(5.4)(5.5).

In the case $k=0$ the wave equation can be reduced to a simple linear equation and the result follows from [16. Thus it is the case $k=-1$ of this theorem which is new. The reduction in the case $k=0$ goes as follows. In that case the field equations imply that $\lambda-\mu+\log t$ is constant in time. It may, however, be dependent on $r$. Suppose that $r$ is replaced by a new coordinate $s$ on the initial hypersurface. Choosing $s$ appropriately makes the transformed quantity $\lambda-\mu+\log t$ constant on the initial hypersurface and hence everywhere. Once this transformation has been carried out the wave equation simplifies to $\ddot{\phi}+t^{-1} \dot{\phi}=\phi^{\prime \prime}$.

Acknowledgements : The authors acknowledge support by a research grant from the VolkswagenStiftung, Federal Republic of Germany. 


\section{References}

[1] H. Andréasson, The Einstein-Vlasov system/kinetic theory. Liv. Rev. Rel. 2002-7.

[2] H. Andréasson, S. Calogero and G. Rein, Global classical solutions to the spherically symmetric Nordström-Vlasov system. Preprint 2003, gr-qc/0311027, To appear in Math. Proc. Camb. Phil. Soc.

[3] H. Andréasson, G. Rein and A. D. Rendall, On the Einstein-Vlasov system with hyperbolic symmetry. Math. Proc. Camb. Phil. Soc. 124, (2003) 529549 .

[4] H. Andréasson, A. D. Rendall and M. Weaver, Existence of CMC and constant areal time foliations in $T^{2}$ symmetric spacetimes with Vlasov matter. Commun. PDE 29, (2004) 237-262.

[5] S. Calogero and H. Lee, The non-relativistic limit of the Nordström-Vlasov system. Commun. Math. Sciences 2, (2004) 19-34.

[6] D. Christodoulou, Bounded variation solutions of the spherically symmetric Einstein-scalar field equations. Commun. Pure Appl. Math. 46, (1993) 11311220.

[7] J. Ehlers, A survey of General Relativity Theory Astrophysics and cosmology, W. Israel (ed.), (1973) 1-125, Reidel, Dordrecht.

[8] A. Douglis, Some existence theorems for hyperbolic systems of partial differential equations in two independent variables. Commun. Pure Appl. Math. $5,(1952)$ 119-154.

[9] K. O. Friedrichs, Nonlinear hyperbolic differential equations for functions of two independent variables. Am. J. Math. 70, (1948) 555-589.

[10] S. Kichenassamy, Nonlinear wave equations. Dekker, New York (1996).

[11] H. Lee, Asymptotic behaviour of the Einstein-Vlasov system with a positive cosmological constant. Preprint 2003, gr-qc/0308035. To appear in Math. Proc. Camb. Phil. Soc.

[12] H. Lee, The Einstein-Vlasov system with a scalar field. Preprint 2004, gr-qc/0404007

[13] G. Rein, The Vlasov-Einstein system with surface symmetry. Habilitation thesis, Munich (1995).

[14] G. Rein, Cosmological solutions of the Vlasov-Einstein system with spherical, plane, and hyperbolic symmetry. Math. Proc. Camb. Phil. Soc. 119, (1996) 739-762. 
[15] A. D. Rendall, Crushing singularities in spacetimes with spherical, plane and hyperbolic symmetry. Class. Quantum Grav. 12, (1995) 1517-1533.

[16] A. D. Rendall, On the nature of singularities in plane symmetric scalar field cosmologies. Gen. Rel. Grav. 27, (1995) 213-221.

[17] A. D. Rendall, Existence of constant mean curvature hypersurfaces in spacetimes with two-dimensional local symmetry. Commun. Math. Phys. 159, (1997) 145-164

[18] A. D. Rendall, The Einstein-Vlasov system. Preprint 2002, gr-qc/0208082

[19] A. D. Rendall, Theorems on existence and global dynamics for the Einstein equations. Liv. Rev. Rel. 2002-6.

[20] A. D. Rendall, Accelerated cosmological expansion due to a scalar field whose potential has a positive lower bound. Class. Quantum Grav 21, (2004) $2445-2454$.

[21] N. Straumann, On the cosmological constant problems and the astronomical evidence for a homogeneous energy density with negative pressure. Preprint 2002, astro-ph/0203330

[22] S. B. Tchapnda N. and N. Noutchegueme, The surface-symmetric EinsteinVlasov system with cosmological constant. Preprint 2003, gr-qc/0304098 To appear in Math. Proc. Camb. Phil. Soc.

[23] S. B. Tchapnda N. and A. D. Rendall, Global existence and asymptotic behaviour in the future for the Einstein-Vlasov system with positive cosmological constant. Class. Quantum Grav. 20, (2003) 3037-3049.

[24] M. Weaver, On the area of the symmetry orbits in $T^{2}$ symmetric spacetimes with Vlasov matter. Class. Quantum Grav. 21, (2004) 1079-1098. 